\title{
Settling velocity of small inertial particles in homogeneous isotropic turbulence from high-resolution DNS
}

\author{
Bogdan Rosa ${ }^{\mathrm{a}, *}$, Hossein Parishani ${ }^{\mathrm{b}}$, Orlando Ayala ${ }^{\mathrm{c}}$, Lian-Ping Wang ${ }^{\mathrm{d}, \mathrm{e}}$ \\ ${ }^{a}$ Institute of Meteorology and Water Management - National Research Institute, \\ 61 Podlesna Street, 01-673 Warsaw, Poland \\ ${ }^{b}$ Department of Earth System Science, University of California, \\ Irvine, CA 92697-3100, USA \\ ${ }^{c}$ Department of Engineering Technology, 214 Kaufman Hall, Old Dominion University, \\ Norfolk, VA 23529, USA \\ ${ }^{d}$ Department of Mechanical Engineering, University of Delaware, \\ Newark, DE 19716-3140, USA \\ ${ }^{e}$ The State Key Laboratory of Coal Combustion, Huazhong University of Science \\ and Technology, Wuhan, 430074, P.R. China
}

\begin{abstract}
The gravitational settling velocity of small heavy particles in a three-dimensional turbulent flow remains a controversial topic. In a homogeneous turbulence of zero mean velocity, both enhanced settling velocity and reduced settling velocity have been reported relative to the still-fluid terminal velocity. Dominant mechanisms for enhanced settling include the preferential sweeping and particleparticle hydrodynamic interactions. The reduced settling could result from loitering (falling particles spend more time in the regions with upward flow), vortex trapping, and drag nonlinearity. Here high-resolution direct numerical simulations (DNS) are used to investigate the settling velocity of non-interacting small heavy particles, for an extended range of flow Taylor microscale Reynolds numbers (up to $R_{\lambda}=500$ ) with varying particle terminal velocity (relative to the Kolmogorov velocity) and particle inertia, by changing the particle-to-fluid density ratio and energy dissipation rate. For the parameter regimes considered here, the preferential sweeping has a dominant effect leading to an increase of
\end{abstract}

\footnotetext{
* Corresponding author

Email address: bogdan.rosa@imgw.com (Bogdan Rosa )
}

Preprint submitted to International Journal of Multiphase Flow

March 4, 2016

(C) 2016. This manuscript version is made available under the Elsevier user license http://www.elsevier.com/open-access/userlicense/1.0/ 
the average settling velocity relative to the terminal velocity; and this increase is mainly governed by particle Froude number (the ratio between the particle inertial response time and the residence time of the particle in a Kolmogorov eddy) and its magnitude depends linearly on the square root of the energy dissipation rate. The reduction of settling due to loitering rarely occurs in a homogeneous turbulence without organized large-scale vortical structures, but is found to emerge only if the particle horizontal motions are blocked (thus removing the preferential sweeping effect), as shown in Good et al. (2014, J. Fluid Mech., 759, R3-1:12). The DNS results were used to develop a parameterization that relates the settling velocity to the particle inertia $(S t)$, Froude number, and $R_{\lambda}$. Finally, sensitivities of the DNS results to the large-scale forcing method and to the drag nonlinearity are also briefly discussed.

Keywords: Settling velocity, inertial particles, cloud droplets, turbulence, DNS

\section{Introduction}

The settling velocity of small heavy particles is relevant to many applications such as pipeline pneumatic transport (Fokeer et al., 2004; Lain and Sommerfeld, 2013), coal combustion (Zhou et al., 2004; Smoot, 2013), transport of biogenic 5 substances in the oceans (Noh et al., 2006), sediment transport in water bodies (Papanicolaou et al., 2008; Keshtpoor et al., 2015), modeling of dust storm (Tsidulko et al., 2002), and warm rain formation (Grabowski and Wang, 2013; Ghosh and Jonas, 2001). More generally, the dynamics of small heavy particles in a turbulent flow affects the spatial distribution of particles (Squires and Eaton, Li et al., 2001), and turbulent collision rate of inertial particles (Zhou et al., 2001; Sundaram and Collins, 1997).

Small heavy particles have a diameter less than the Kolmogorov scale of the carrier fluid turbulence and a density much larger than that of the fluid. These 
$S t$ (the ratio of their inertial response time $\tau_{p}$ to the flow Kolmogorov time $\left.\tau_{K}\right)$, and a significant terminal velocity, in terms of the ratio $S_{V}=V_{T} / v_{K}$ with $V_{T}$ being the particle still-fluid terminal velocity and $v_{K}$ the flow Kolmogorov velocity. In this work, we concern mainly the average settling velocity of such particles in a homogeneous turbulent flow, under the assumption that the particle mass loading is very low so that the effect of particles on the fluid turbulence is weak (i.e., one-way coupling) and particle-particle local hydrodynamic interactions can be neglected. It is well known that the average settling velocity, relative to the mean flow, may differ from $V_{T}$ for a variety of reasons, to be discussed below.

The motion of small heavy particles depends on two governing parameters which can be expressed in terms of other physical parameters as follows (Wang et al., 2006):

$$
S t=\frac{\tau_{p}}{\tau_{K}}=\frac{2}{9} \frac{\rho_{p}}{\rho_{f}} \frac{\varepsilon^{0.5}}{\nu^{1.5}} a^{2}, \quad S_{V}=\frac{V_{T}}{v_{K}}=\frac{2}{9} \frac{\rho_{p}}{\rho_{f}} \frac{g}{\varepsilon^{0.25} \nu^{1.25}} a^{2},
$$

which implies that, in real applications, there are five physical parameters affecting the dynamics: the particle radius $a$, the gravitational acceleration $\mathrm{g}$, the energy dissipation rate $\varepsilon$, the fluid kinematic viscosity $\nu$ and the particle-to-fluid density ratio $\sigma=\rho_{p} / \rho_{f}$.

An important derived parameter combining $S t$ and $S_{V}$ is the particle Froude number, namely, the ratio of particle response time $\tau_{p}$ to the residence time of the particle in a Kolmogorov eddy (Davila and Hunt, 2001)

$$
F r=\frac{\tau_{p}}{\Gamma_{\text {vort }} / v_{p}^{2}}=\frac{\tau_{p} V_{T}^{2}}{\eta v_{K}}=S t S_{V}^{2}=\frac{\tau_{p}^{3} g^{2}}{\nu}=\left(\frac{2}{9}\right)^{3}\left(\frac{\rho_{p}}{\rho_{f}}\right)^{3} \frac{g^{2} a^{6}}{\nu^{4}},
$$

which is independent of flow energy dissipation rate. Here, $\eta$ is the Kolmogorov length scale. For water droplets in air, this parameter reaches one when $a \approx$ $20 \mu \mathrm{m}$. Ayala et al. (2008) showed that the sedimenting particles obtain a maximum relative settling-velocity enhancement at $a \approx 20 \mu \mathrm{m}$ (see also Table 4

40 in Section 3.1). Another parameter affecting the level of settling velocity is the flow Reynolds number $\left(R_{\lambda}\right)$ which defines the range of flow length scales or the large-to-small scale ratio in the flow. $R_{\lambda}=u^{\prime} \lambda / \nu$ where $u^{\prime}$ is the rms fluctuating 
velocity and $\lambda$ is the transverse Taylor microscale. The effects of key parameters on the dimensionless parameters are shown in Table 1

Table 1: Relations between dimensional and nondimensional parameters.

\begin{tabular}{lllll}
\hline \hline & $S t$ & $S_{V}$ & $F r$ & $R_{\lambda}$ \\
\hline$a$ & $S t \propto a^{2}$ & $S_{V} \propto a^{2}$ & $F r \propto a^{6}$ & no effect \\
$\mathrm{g}$ & no effect & $S_{V} \propto \mathbf{g}$ & $F r \propto \mathbf{g}^{2}$ & no effect \\
$\varepsilon$ & $S t \propto \varepsilon^{0.5}$ & $S_{V} \propto \varepsilon^{-0.25}$ & no effect & $R_{\lambda} \propto \varepsilon^{-0.5}$ \\
$\nu$ & $S t \propto \nu^{-1.5}$ & $S_{V} \propto \nu^{-1.25}$ & $F r \propto \nu^{-4}$ & $R_{\lambda} \propto \nu^{-0.5}$ \\
$\sigma=\frac{\rho_{p}}{\rho_{f}}$ & $S t \propto \sigma$ & $S_{V} \propto \sigma$ & $F r \propto \sigma^{3}$ & no effect \\
$N$ & no effect & no effect & no effect & $R_{\lambda} \propto N^{2 / 3}$ \\
\hline \hline
\end{tabular}

Based on the simplified equation of motion we assume that the difference between average settling velocity $\left\langle V_{S}\right\rangle$ and the terminal velocity $V_{T}$ is an unknown function of the following parameters

$$
\left\langle V_{S}\right\rangle-V_{T}=\mathbf{F}\left(\tau_{p}, V_{T}, \varepsilon, u^{\prime}, L_{f}, \ldots\right)
$$

where, $L_{f}$ is the integral lengthscale of the turbulent flow. Since $L_{f} \sim \eta R_{\lambda}^{1.5}$ and $R_{\lambda}=\sqrt{15}\left(u^{\prime} / v_{K}\right)^{2}$ the integral lengthscale can be omitted from Eq. 3 50 The dimensional analysis leads to three equivalent representations of the Eq. 3 . namely

$$
\begin{aligned}
& \frac{\left\langle V_{S}\right\rangle-V_{T}}{v_{K}}=\mathbf{F}_{1}\left(S t, S_{V}, R_{\lambda}, \ldots\right), \\
& \frac{\left\langle V_{S}\right\rangle-V_{T}}{V_{T}}=\mathbf{F}_{2}\left(S t, S_{V}, R_{\lambda}, \ldots\right)=\frac{\mathbf{F}_{1}}{S_{V}} \\
& \frac{\left\langle V_{S}\right\rangle-V_{T}}{u^{\prime}}=\mathbf{F}_{3}\left(S t, S_{V}, R_{\lambda}, \ldots\right)=\frac{15^{0.25}}{R_{\lambda}} \mathbf{F}_{1} .
\end{aligned}
$$

In each of these three equations, the change in the settling rate can be expressed in terms of the same function $\mathbf{F}_{1}$. This function, depends only on the dimensionless parameters such as $S t, S_{V}$ (or $F r$ ), and $R_{\lambda}$. 
In a homogeneous turbulence of zero mean velocity, both enhanced settling velocity and reduced settling velocity have been reported. The dominant mechanism for enhanced settling velocity of small heavy particles in a turbulent flow is the preferential sweeping (Wang and Maxey, 1993). In their pioneering work, Maxey and Corrsin (1986) demonstrated that inertial particles can distribute very non-uniformly in a steady non-uniform flow, and consequently sedimenting particles may converge to a path located on the downward side of a vortex, leading to a higher settling velocity. Maxey (1987) extended the above study to a time-dependent non-uniform flow, and found both analytically and numerically that inertial particles accumulate in regions of low vorticity and high strain rate, a phenomenon now known as the preferential concentration. He found that the preferential concentration leads to a bias of particle trajectory and an enhanced settling rate. Wang and Maxey (1993) performed direct numerical simulations to show that the preferential concentration is strongest when $S t$ and $S_{V}$ are of the order one in a realistic turbulent flow. They also demonstrated that the mean particle settling velocity is significantly enhanced under similar conditions, with the level of enhancement depending on the large-scale flow statistics.

Fung (1997) modeled the motion of small spherical particles in an infinite, two-dimensional unsteady flow. He showed that enhancement of the settling velocity occurs when $V_{T} / \sigma_{u} \leq 0.7$. Here $\sigma_{u}$ is a characteristic velocity of the 75 flow. The maximum increase occurs when $V_{T} \approx 0.5 \sigma_{u}$. In the steady flow (flow with one length scale), however, the total suspension takes place when $V_{T}$ is of order of the characteristic fluid velocity. Lillo et al. (2008) studied the settling velocity of heavy particles in two-dimensional turbulent and laminar flows. They showed that at large flow velocities particles are effectively guided to the downflow regions and the actual settling velocity is larger than $V_{T}$. Afonso (2008) investigated the settling of inertial particles in 2D cellular flow. He found that for square, static cellular flows and at relatively small $S t$ the settling velocity, is larger than the terminal velocity. However, starting from a certain critical value of $S t$, the falling velocity becomes lower than $V_{T}$. Although the author does not 85 state explicitly what mechanism reduces the settling velocity, we assume that 
the reduction may be due to vortex trapping.

Using both DNS and large-eddy simulation (LES), Yang and Lei (1998) examined the role of turbulent scales in the settling velocity of heavy particles. They found that large-scale flow plays a significant role in determining the increase in settling velocity, namely, the increase in settling scales with rms fluid velocity $\left(u^{\prime}\right)$, not $v_{K}$, but peaks at $S t \approx 1$.

The problem of settling rate of inertial particles has also been investigated experimentally. Zhou and Cheng (2009) monitored the settling velocity of lowinertia solid particles in turbulent water flow generated by an oscillating grid. They found that the actual settling velocity of the particles is generally smaller than the corresponding terminal velocity. The authors showed that the reduced settling velocity correlates with the vertical velocity fluctuation. Ruiz et al. (2004) measured the settling velocity of phytoplankton cells in turbulent flows generated in water by different mechanical devices. They concluded that the larger settling velocity observed in the experiment is likely due to mechanism of preferential sweeping. Cuthbertson and Ervine (2007) investigated the behavior of fine sand particles in turbulent open channel flow generated over rough, porous bed condition. Based on the experimental data they concluded that the settling rate of the suspended fine sand can be significantly enhanced over the terminal velocity.

Other mechanisms for enhanced settling, discussed in the literature, include the influence of local particle-particle hydrodynamics interactions (Aliseda et al., 2002; Alipchenkov and Zaichik, 2009) and the effect of two-way coupling (Dejoan, 2011; Yang and Shy, 2005; Bosse et al., 2006).

There are also a number of turbulence-induced mechanisms that lead to reduction of particle settling velocity. The first concerns the effect of nonlinear drag. This mechanism has been investigated numerically in several previous studies. A first rigorous numerical simulation was developed by Wang and Maxey (1993). Their DNS were limited only to small turbulent Reynolds numbers and to three particle Stokes numbers. They found that the drag nonlinearity slightly reduces the net increase in the settling velocity. 
In another study, Mei (1994) showed that for a nonfrozen turbulence the nonlinear drag increases the effective particle time constants and this leads to an effective reduction in the settling velocity. Similar conclusions result from the study by Fung (1993) in which a turbulent flow is modeled using kinematic simulations (KS). In a subsequent study, Chan and Fung (1999) investigated the gravitational settling of small inertial particles in a simple, two-dimensional periodic cellular flow. They found that the settling velocity of inertial particles in such a flow is larger than the terminal velocity by $120 \%$ (assuming $V_{T} / \sigma_{u}<$ $0.5)$. It should be added that their particle equation of motion included the square drag. The effect of nonlinear drag on the particle settling velocity has also been examined by Stout et al. (1995). They demonstrated that reduction in the average settling velocity can be greater than $35 \%$, and the rate of reduction decreases with decreasing particle Reynolds number. The main cause here is the decreasing drag nonlinearity and the reduction vanishes within the Stokes drag regime.

The results presented by Mei (1994), Fung (1993) and Stout et al. (1995) are in contrast to those developed by Wang and Maxey (1993) and Chan and Fung (1999). The discrepancy may be caused by the differences in the numerical approach employed to simulate the turbulent flows. In the literature, there are also some studies stating that the impact of the nonlinear drag on the settling velocity is negligible. For example, Nielsen (1993) derived an analytical formula that relates the maximum fluid acceleration to the reduction in the settling velocity. Based on this formulation and experimental measurements, Nielsen concluded that the effect of nonlinear drag on the settling velocity is less than $10^{-5} V_{T}$ and hence has no practical importance.

The second mechanism for reduced settling is the vortex trapping. Tooby et al. (1977) showed experimentally that forced vortices with horizontal axes can trap bubbles and heavy particles and thus eliminate their settling velocity completely. Manton (1974) derived equations of motion for particles moving through an axisymmetric eddy with a horizontal axis. Based on this analysis, Manton claimed that the turbulence should cause particles to fall at much lower rate than their 
terminal velocity, even in the absence of a mean updraft. Nielsen (1993) proposed a basic theoretical model to explain the mechanism of vortex trapping. Vilela and Motter (2007) examined the trapping mechanism of aerosol particles in two idealized open flows such as the blinking vortex system and the leepfrog system. Their numerical simulations showed that for a broad range of conditions the permanent trapping of the inertial particles is possible even when the gravitational effect is large. Pasquero et al. (2003) investigated the settling of heavy particles in a steady two-dimensional random velocity field. Their analytical considerations show that particle suspension takes place when small-scale turbulence suitably alters the curvature of the streamlines around an eddy. They also argued that when the flow is unsteady, permanent suspension of particles becomes very unlikely. The above flows are perhaps not strictly relevant to $3 \mathrm{D}$ homogeneous isotropic turbulence, and therefore the latter results may not be directly generalized to 3D turbulence.

The third reduced-settling mechanism is known as the loitering effect. This mechanism was first observed and described by Nielsen (1993). Nielsen observed that in a relatively weak turbulence the settling velocity of heavy particles is reduced by $20-40 \%$. He argued that this delaying effect results from the fact that fast-falling particles spend more time in the regions with the upward flow. Such particles cannot be trapped by eddies but also cannot be effectively guided along vortical structures. This finding was confirmed in subsequent laboratory experiments by Kawanisi and Shiozaki (2008) and Cadiergue et al. (1999). Good et al. (2014) claimed that this effect is not observed in DNS simulations when the Stokes drag is assumed.

In this study, we employ direct numerical simulations to investigate the settling velocity of non-interacting small heavy particles. The first objective is to explore how the settling velocity varies with the non-dimensional parameters, 175 namely $S t, S_{V}, F r, R_{\lambda}, \sigma$ and turbulence intensity (i.e. $u^{\prime}$ ) or equivalently the energy dissipation rate $(\varepsilon)$ at fixed $R_{\lambda}$ and kinematic viscosity. To a limited extent, similar studies have been carried out in the past (see comparison in Table 21). 
Table 2: Overview of previous DNS studies investigating the settling velocity of small inertial particles in homogeneous isotropic turbulence. The table compares parameter setting and average flow statistics. E/R refers to the nature of obtained results, namely (E)nhancement or (R)eduction of the settling rate (B - both).

\begin{tabular}{|c|c|c|c|c|c|c|c|c|c|c|}
\hline Study & $d_{p} / \eta$ & St & $S v$ & $\mathrm{Fr}$ & $\rho_{p} / \rho_{f}$ & Coupling & $\begin{array}{r}R_{\lambda} \\
\text { up to }\end{array}$ & $N$ & $\begin{array}{l}\text { Nonlin. } \\
\text { drag }\end{array}$ & $\mathrm{E} / \mathrm{R} / \mathrm{B}$ \\
\hline Wang and Maxev (1993) & $0.01-0.2$ & $0.2-3.25$ & $0.2-3.25$ & $0.008-34.33$ & 1000 & 1-way & 61.5 & 64 & Yes & $\mathrm{E}$ \\
\hline Yang and Lei (1998) & - & $0.1-2.5$ & $0.1-3.5$ & $0.001-30.625$ & 1000 & 1-way & 65.3 & 96 & Yes & $\mathrm{E}$ \\
\hline Bosse et al. (2006) & - & $\sim 1$ & $\sim 1$ & $\sim 1$ & 5000 & 2-way & 42.7 & 64 & No & $\mathrm{E}$ \\
\hline Avala et al. (2008) & $0.011-0.101$ & $0.032-2.282$ & $0.626-12.516$ & $0.0124-357.48$ & 1000 & 1-way & 72.4 & 128 & No & $\mathrm{E}$ \\
\hline Dejoan (2011) & $0.036-0.085$ & $\sim 1$ & $\sim 1$ & $\sim 1$ & 5000 & 2-way & 130 & 512 & No & $\mathrm{E}$ \\
\hline Good et al. (2014) & $0.012-0.55$ & $0.03-60.0$ & $0.1-100$ & $0.0003-600,000$ & 877 & 1-way & 227 & 512 & Yes & B \\
\hline Present study & $0.013-4.02$ & $0.007-8.97$ & $0.355-63.1$ & $0.0002-12,618$ & $500-10,000$ & 1-way & 500 & 1024 & Yes & $\mathrm{E}$ \\
\hline
\end{tabular}


The second objective is to develop a parameterization (analytical formula)

$$
\begin{aligned}
& \text { that relates the settling velocity to the set of non-dimensional parameters in- } \\
& \text { dicated in Eq. 4. The third objective is to quantify how the large-scale forc- }
\end{aligned}
$$
ing scheme, gravitational acceleration and the drag nonlinearity influence the settling statistics. Finally, the fourth objective is to explore the possible mechanisms by which the contributing parameters alter the particle sedimentation velocity.

The remainder of the paper is organized as follows. In Section 2, we describe the essential details of the numerical method. Results from the DNS are discussed in Section 3. These include sensitivity of the settling rate to $S t, S_{V}$, $F r, \sigma, \varepsilon, u^{\prime}, R_{\lambda}$, large-scale forcing scheme, gravitational acceleration, and the drag nonlinearity. Numerical experiments with artificially forced loitering are discussed in Section 4. Section 5 contains a summary and main conclusions.

\section{Methodology}

The turbulent fluid flow is simulated by a standard pseudo-spectral method (Orszag and Patterson, 1972), which solves the Navier-Stokes equation on a 3D uniform mesh with $N$ equally spaced grid points in each spatial direction. The flow domain is a cube with size $2 \pi$. Periodic boundary conditions are enforced which is consistent with the 3D discrete Fourier transform applied to the fluid velocity field.

The fluid velocity $\mathbf{U}(\mathbf{x}, t)$ is obtained by numerical integration of the rotational form of the Navier-Stokes equation

$$
\frac{\partial \mathbf{U}}{\partial t}=\mathbf{U} \times \omega-\nabla\left(\frac{P}{\rho}+\frac{1}{2} \mathbf{U}^{2}\right)+\nu \nabla^{2} \mathbf{U}+\mathbf{f}(\mathbf{x}, t) .
$$

for an incompressible fluid satisfying the continuity equation

$$
\nabla \cdot \mathbf{U}(\mathbf{x}, t)=0
$$

Here $\omega \equiv \nabla \times \mathbf{U}$ is the vorticity, $P$ is the pressure, $\rho$ is fluid density and $\nu$ is fluid kinematic viscosity. 
A stationary turbulence is maintained by the forcing term $\mathbf{f}(\mathbf{x}, t)$ which is nonzero only for a few low-wave-number modes $(|k|<\sqrt{8})$ in the Fourier space. We implemented two forcing methods allowing us to study the sensitivity of the results on the forcing method (as discussed by Rosa et al. (2015)). The details of the two forcing methods are described in Section 3.4.

We first evolve the flow (without particles) from $t=0$ up to at least $t=$ $10 T_{e}$ ( $T_{e}$ is the large-eddy turnover time) to ensure that the flow is statistically stationary. This eliminates any effect of initial flow condition on the statistics and dynamics of the resulting stationary homogeneous and isotropic turbulence. Detailed flow statistics obtained at different grid resolutions and different forcing mechanisms are presented in our preceding article (Rosa et al., 2013). Here, 215 in Table 3 we only present a few basic parameters, namely, the kinematic fluid viscosity $\nu$, the energy dissipation rate $\varepsilon$, the Kolmogorov length $\eta$, the Kolmogorov time $\tau_{K}$, the eddy turnover time $T_{e}$, and the rms fluid velocity $u^{\prime}$. Since the mean flow velocity in the domain is zero, $u^{\prime}$ defines also the turbulence intensity. The spatial resolution of the simulations was monitored by $k_{\max } \eta$. 220 In pseudo-spectral simulations this value should be greater than unity for fine scales to be resolved. In order to maximize turbulent Reynolds number $\left(R_{\lambda}\right)$ we kept $k_{\max } \eta$ close to unity for every mesh size and for both forcing methods (see Table 3).

The motion of heavy inertial particles is tracked by a Lagrangian approach. 225 Once the background turbulent velocity field $\mathbf{U}(\mathbf{X}, t)$ is established, inertial particles are advanced by solving their equation of motion (Maxey and Riley, 1983) including particle inertia, viscous drag, and the body force

$$
\begin{gathered}
\frac{d \mathbf{V}^{(k)}(t)}{d t}=-f\left(R e, V_{r e l}\right) \frac{\mathbf{V}^{(k)}(t)-\mathbf{U}\left(\mathbf{Y}^{(k)}(t), t\right)}{\tau_{p}^{(k)}}+\mathbf{g} \\
\frac{d \mathbf{Y}^{(k)}(t)}{d t}=\mathbf{V}^{(k)}(t) .
\end{gathered}
$$

Here $k$ is the particle number, $\tau_{p}^{(k)}$ is the Stokes inertial response time, $\mathbf{V}^{(k)}(t)$ is the particle velocity and $\mathbf{U}\left(\mathbf{Y}^{(k)}(t), t\right)$ denotes the fluid velocity at the particle 
Table 3: Parameters and realized statistics (in spectral units) at the statistically stationary stage of the simulated flows using both deterministic and stochastic forcing schemes.

\begin{tabular}{lrrrrrr}
\hline \hline$N$ & 32 & 64 & 128 & 256 & 512 & 1024 \\
\hline & \multicolumn{5}{c}{ Deterministic forcing scheme } \\
\cline { 2 - 7 }$\nu \times 10^{4}$ & 170 & 67 & 28 & 11 & 4.8 & 1.8 \\
$\varepsilon \times 10^{1}$ & 1.52 & 1.79 & 2.08 & 2.00 & 1.91 & 1.96 \\
$\eta \times 10^{2}$ & 7.553 & 3.604 & 1.805 & 0.9033 & 0.4912 & 0.2339 \\
$\tau_{K} \times 10^{1}$ & 3.357 & 1.939 & 1.164 & 0.7420 & 0.5030 & 0.3040 \\
$T_{e}$ & 3.811 & 3.848 & 3.636 & 3.774 & 3.946 & 3.917 \\
$u^{\prime} \times 10^{1}$ & 7.58 & 8.28 & 8.67 & 8.68 & 8.65 & 8.73 \\
$k_{\text {max }} \eta$ & 1.095 & 1.099 & 1.128 & 1.143 & 1.250 & 1.194 \\
\hline & & & Stochastic forcing scheme & \\
$\nu \times 10^{3}$ & 450 & 200 & 75 & 28 & 12 & 4.5 \\
$\varepsilon$ & 3334 & 3449 & 3504 & 3673 & 3670 & 3751 \\
$\eta \times 10^{2}$ & 7.244 & 3.909 & 1.867 & 0.8810 & 0.4666 & 0.2223 \\
$\tau_{K} \times 10^{2}$ & 1.167 & 0.7646 & 0.4651 & 0.2774 & 0.1815 & 0.1099 \\
$T_{e} \times 10^{2}$ & 8.362 & 9.531 & 10.14 & 10.3 & 10.6 & 8.444 \\
$u^{\prime}$ & 16.65 & 18.07 & 18.76 & 19.36 & 19.61 & 19.85 \\
$k_{\text {max }} \eta$ & 1.050 & 1.192 & 1.167 & 1.114 & 1.188 & 1.135 \\
\hline \hline
\end{tabular}

location $\mathbf{Y}^{(k)}(t)$, Re is particle Reynolds number, $V_{\text {rel }}$ is particle-fluid relative velocity and $\mathbf{g}$ is the gravitational acceleration. $f$ is the drag coefficient which in this paper is set to $f=1$ except in Section 3.6 for which a non-linear drag is introduced and discussed. Particles are initially introduced to the flow at random locations. In this study we focus mainly on inertial particles relevant to cloud droplets of radius from 10 to $60 \mu \mathrm{m}$.

A complete description of the code together with results of the numerical experiments and scalability analysis can be found in (Avala et al., 2014). In this 
study, we consider only the one-way coupling, i.e. the fluid flow is not affected by the presence of particles.

Our DNS implementation is validated in two series of simulations performed at low Taylor microscale Reynolds number $\left(R_{\lambda}=27.8\right.$ and 48.4). The setup of the experiments follows the study by Wang and Maxey (1993). For this validation, it is assumed that the terminal velocity of the particles is equal to the Kolmogorov velocity $\left(S_{V}=1\right)$. The focus is on computing the settling velocity of small rigid spherical particles with the Stokes number ranging from 0.2 to 4 . In Fig. 1 we compare results from present DNS with results reported

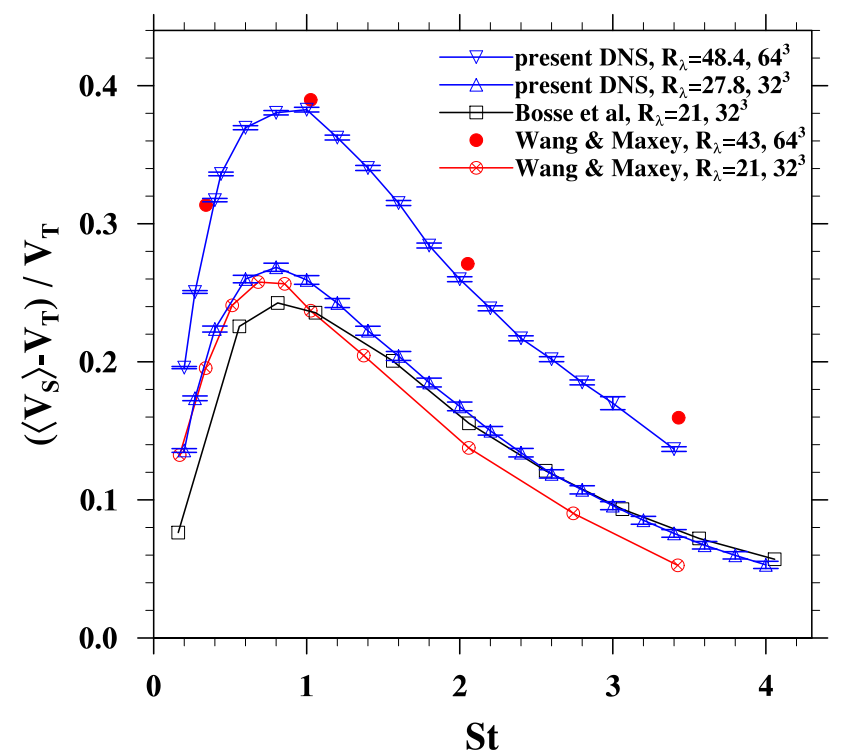

Figure 1: The increase $\left(\left\langle V_{S}\right\rangle-V_{T}\right)$ in the particle mean settling velocity normalized by the terminal velocity as a function of the Stokes number. The simulations have been performed assuming $V_{T} / v_{K}=1$. The results from present DNS simulations (blue color) are compared with results developed by Wang and Maxey (1993) and Bosse et al. (2006).

in (Wang and Maxey, 1993; Bosse et al., 2006). The figure shows an increase in the particle mean settling velocity normalized by terminal velocity for different values of the Stokes number. We conclude that the present results are consistent with the results reported in (Wang and Maxey, 1993; Bosse et al., 2006). The small differences at low grid resolution $32^{3}$ are associated with the differences 
in $R_{\lambda}$ and also the details of large-scale flow forcing.

\section{The results of numerical simulations}

3.1. Settling velocity of inertial particles relevant to cloud droplets in turbulent flow with different $\varepsilon$

In the first series of numerical experiments, we assume that the particles are water droplets of density $\rho_{p}=1 \mathrm{~g} / \mathrm{cm}^{3}$. The particles move in a turbulent air of density $\rho_{f}=0.001 \mathrm{~g} / \mathrm{cm}^{3}$ and kinematic viscosity $\nu=0.17 \mathrm{~cm}^{2} / \mathrm{s}$. The vertical downward acceleration is set to $\mathbf{g}=9.8 \mathrm{~m} / \mathrm{s}^{2}$ and the average energy dissipation rate of the turbulent flow is in the range of 10 to $1000 \mathrm{~cm}^{2} / \mathrm{s}^{3}$. Such a setting is typical for the atmospheric cloud processes (Siebert et al., 2006). The setting can also be described in terms of three non-dimensional parameters $\left(F r=S t S_{V}^{2}\right.$ is not an independent parameter) specified in Table 1 . The Taylor microscale Reynolds number is constant $\left(R_{\lambda}=143.74\right)$ for each simulation. The values of these dimensionless parameters for three different energy dissipation rates are listed in Table 4

Since both $R_{\lambda}$ and the fluid viscosity $\nu$ are fixed in the simulations, the relation between energy dissipation rate and turbulence intensity is uniquely defined by

$$
u^{\prime}=(\varepsilon \nu)^{0.25} \frac{\sqrt{R_{\lambda}}}{15^{0.25}} \propto \varepsilon^{1 / 4}
$$

Hence the dependence of the settling velocity on the dissipation rate can be equivalently expressed as dependence on the turbulence intensity $\left(u^{\prime}\right)$.

Results from similar numerical simulations, but limited to low turbulent Reynolds numbers $\left(R_{\lambda} \leq 72.4\right)$ and to two energy dissipation rates (100 and $400 \mathrm{~cm}^{2} / \mathrm{s}^{3}$ ) have been reported in the study by Avala et al. (2008). The present simulations were performed at a finer grid resolution of $256^{3}$ and consequently, the Taylor microscale Reynolds number of the present DNS is approximately twice larger $\left(R_{\lambda}=143.74\right)$ than those of Avala et al. (2008). Additionally, the numerical uncertainty of the current results is smaller than those presented in (Ayala et al., 2008). We run the DNS longer than $10 T_{e}$ with up to one million 
Table 4: Basic properties of cloud droplets

\begin{tabular}{|c|c|c|c|c|c|c|c|}
\hline \multirow[b]{2}{*}{$a[\mu \mathrm{m}]$} & \multicolumn{2}{|c|}{$\varepsilon=10 \mathrm{~cm}^{2} / \mathrm{s}^{3}$} & \multicolumn{2}{|c|}{$\varepsilon=400 \mathrm{~cm}^{2} / \mathrm{s}^{3}$} & \multicolumn{2}{|c|}{$\varepsilon=1000 \mathrm{~cm}^{2} / \mathrm{s}^{3}$} & \multirow[b]{2}{*}{$F r$} \\
\hline & $S t$ & $S_{V}$ & $S t$ & $S_{V}$ & $S t$ & $S_{V}$ & \\
\hline 10 & 0.010 & 1.12 & 0.063 & 0.45 & 0.100 & 0.355 & 0.013 \\
\hline 15 & 0.023 & 2.52 & 0.143 & 1.00 & 0.226 & 0.798 & 0.144 \\
\hline 20 & 0.040 & 4.49 & 0.254 & 1.78 & 0.401 & 1.419 & 0.808 \\
\hline 22.5 & 0.051 & 5.68 & 0.321 & 2.26 & 0.507 & 1.796 & 1.64 \\
\hline 27.5 & 0.076 & 8.48 & 0.479 & 3.37 & 0.758 & 2.683 & 5.46 \\
\hline 30 & 0.090 & 10.1 & 0.571 & 4.01 & 0.902 & 3.193 & 9.20 \\
\hline 40 & 0.160 & 17.9 & 1.014 & 7.14 & 1.604 & 5.676 & 51.7 \\
\hline 50 & 0.251 & 28.0 & 1.585 & 11.1 & 2.506 & 8.869 & 197 \\
\hline 60 & 0.361 & 40.4 & 2.283 & 16.0 & 3.609 & 12.77 & 589 \\
\hline
\end{tabular}

particles. The long simulation time and the large number of particles resulted in the small statistical uncertainty.

The effect of turbulence on the settling rate is quantified by comparing the settling velocities of particles in turbulent flow against those in a stagnant air (i.e. terminal velocity). Fig. 2 shows the increase in the particle settling velocity normalized by the terminal velocity as a function of both particle (water droplet) radius and non-dimensional parameter Fr. Different lines correspond to simulations performed with different value of energy dissipation rate (equivalently $\left.u^{\prime}\right)$.

Several conclusions can be drawn from Fig. 2. First, the settling velocity of low inertia particles tends to zero, consistent with the tracer limit in which particles behave like fluid elements. We note that the mean flow velocity in the domain is zero. Second, the maximum increase in the settling velocity is observed at Froude number equal to 1 and is independent of the energy dissipation rate, consistent with the theory of Davila and Hunt (2001) and the 295 results of Avala et al. (2008). Third, the dynamics of droplets with large $S_{V}$ 


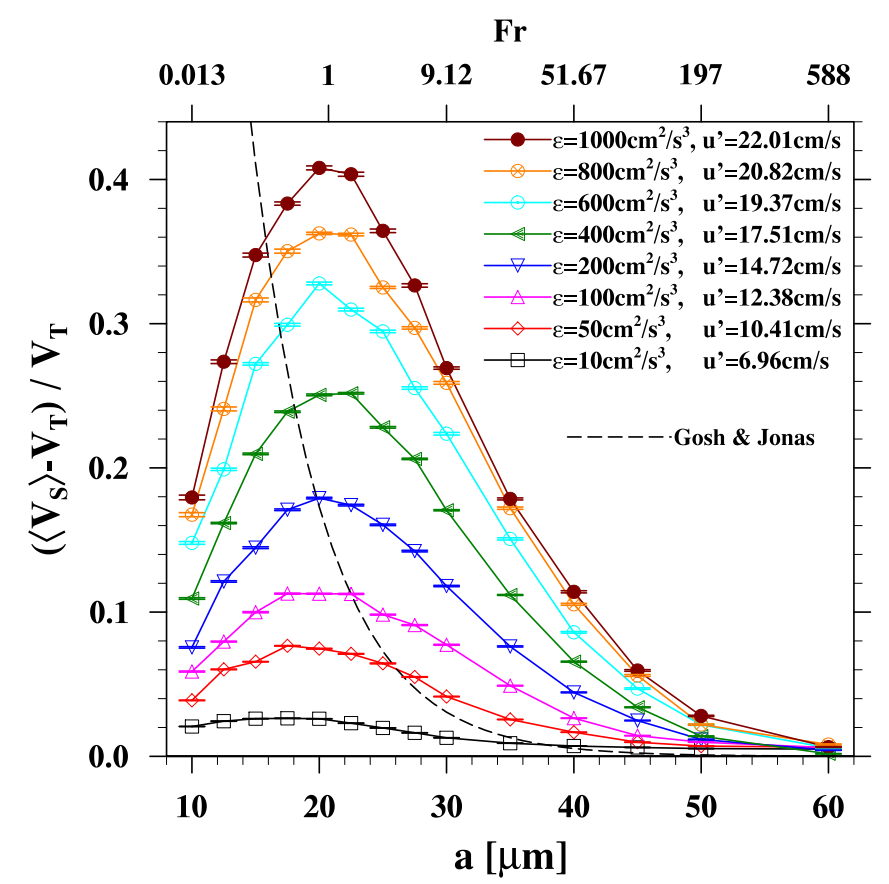

Figure 2: The increase in settling velocity of inertial particles in turbulent air normalized by their terminal velocity. Different colors correspond to simulations with different energy dissipation rate or equivalently turbulence intensity. For each series of simulations at fixed $\varepsilon$, the maximal increase of velocity occurs at $F r \approx 1$. All simulations were performed at the grid resolution of $256^{3}\left(R_{\lambda}=143.74\right)$ using the stochastic forcing.

$\left(r>60 \mu \mathrm{m}, S_{V}>10\right)$ is weakly modified by turbulence due to very short interaction time with turbulent eddies $(F r>500)$. Therefore, for these particles the settling velocity is comparable with their terminal velocity. Also in Fig. 2 the empirical formula of Ghosh and Jonas (2001) is plotted for comparison. Their model predicts the amplification effect as $\left(\left\langle V_{S}\right\rangle-V_{T}\right) / V_{T}=\alpha^{-\beta a}$, where $\alpha=5.5$ and $\beta=0.173 \mu \mathrm{m}^{-1}$. The differences between DNS and the empirical formula are large. Even the trend for large inertia particles is only roughly predicted. The empirical formula was developed by curve-fitting an exponential function to a few discrete data points computed based on the theoretical model. Therefore, this function cannot correctly represent amplification effect both for small and large droplets. 
The DNS results from Fig. 2 can be presented equivalently as a function of two other nondimensional parameters, namely $S t$ and $S_{V}$. The contour plot in Fig. 3 shows the increase in the settling velocity normalized by $u^{\prime}$ as a function of both $\ln S t$ and $\ln S_{V}$. The black circles represent the data points from various simulations and the two-dimensional distribution is obtained by spatial interpolation. Compared to the previous figure, Fig. 3 shows also results from two additional series of simulation performed at $\varepsilon=5$ and $30 \mathrm{~cm}^{2} / \mathrm{s}^{3}$.

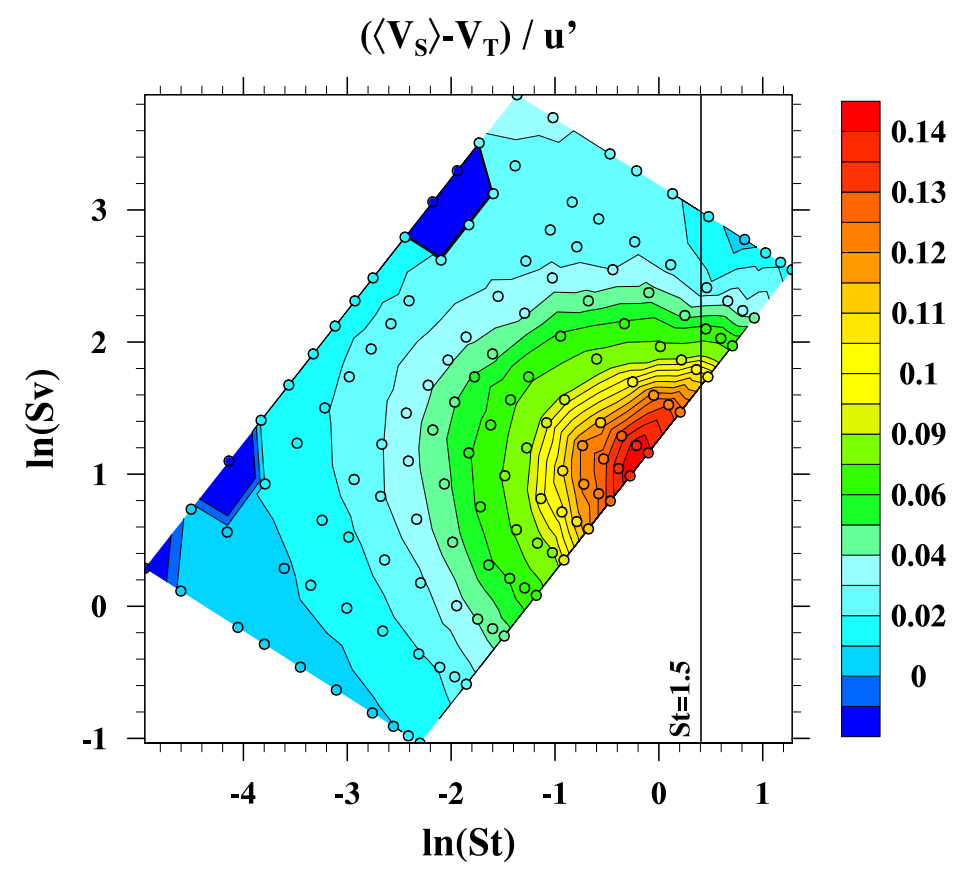

Figure 3: Difference between the average settling velocity of inertial particles relevant to cloud droplets in turbulent flow and its terminal velocity in stagnant air normalized by $u^{\prime}$. This difference is presented as a function of $\ln (S t)$ and $\ln \left(S_{V}\right)$.

Only in two cases, we observed a reduction of the settling velocity. For particles with low inertia of $S t=0.007$ and small settling of $S_{V}=1.33$ the reduction is about $0.18 \mathrm{~mm} / \mathrm{s}$ while for particles with $S t=0.143$ and larger $S_{V}=27.01$ the observed reduction is $4 \mathrm{~mm} / \mathrm{s}$. These values are relatively small and are comparable in magnitude with the statistical uncertainty. We note that the 
results from present DNS in the range $S t>1.5$ differ from those obtained experimentally by Yang and Shy (2003), namely, reduction of the settling velocity is not observed in DNS data. Yang and Shy measured the settling velocity of heavy tungsten and glass particles in an aqueous near-isotropic turbulence. They stated, that the settling velocity of particles with $S t>1.5$ is smaller than their terminal velocity. This reduction results from the nonlinear drag effect due to both larger particle Reynolds number and interaction with larger turbulent scales. Yang and Shy (2003) observed the reduction in $\left\langle V_{S}\right\rangle$ only for glass particles with a large particle Reynolds number $R e=\left(2 a V_{T} / \nu\right)=39$. In our DNS, however, the maximal $R e$ is significantly lower at $R e=15.07$.

A similar conclusion is reported in a recent study by Good et al. (2014). 330 Good et al. (2014) performed experiments with water droplets in turbulent air in which the turbulence was generated using 32 loudspeaker jets. Their experiments show that for certain particle classes there is a reduction of the settling velocity. They also concluded that such a reduction is not observed in DNS results when the particles are subjected to the linear drag.

All DNS discussed in this section were performed at the same grid resolution ( $N=256)$. Thus, both $R_{\lambda}$ and $u^{\prime} / v_{K}$ are fixed for each simulation. Therefore, the changes in the energy dissipation rate can be viewed equivalently as changes in the particle inertia. To confirm this interdependence, the original data from Fig. 2 needs to be presented in another nondimensional form. Fig. 4 shows Stokes number versus the particle Froude number. With such scaling all the lines collapse to one curve. This normalization turns out to be helpful in developing a useful parameterization. Based on Eq. 4. we postulate that the increase in the settling velocity takes on the following form

$$
\frac{\left\langle V_{S}\right\rangle-V_{T}}{V_{T}}=\frac{\mathbf{F}_{1}\left(S t, S_{V}, R_{\lambda}, \ldots\right)}{S_{V}}=S t \cdot \mathbf{F}_{4}(F r) \cdot F^{m\left(R_{\lambda}\right)} \cdot \mathbf{F}_{5}\left(R_{\lambda}\right)
$$

345 where the product of $\operatorname{Fr}^{m\left(R_{\lambda}\right)}$ and $\mathbf{F}_{5}\left(R_{\lambda}\right)$ is equal to unity at $R_{\lambda}=143.74$. In other words, we decompose the function $\mathbf{F}_{1}\left(S t, S v, R_{\lambda}\right)$ from Eq. 4 into two parts (i.e. $\mathbf{F}_{4}$ and $\mathbf{F}_{5}$ ), the first of which depends only on $F r$, and the second 


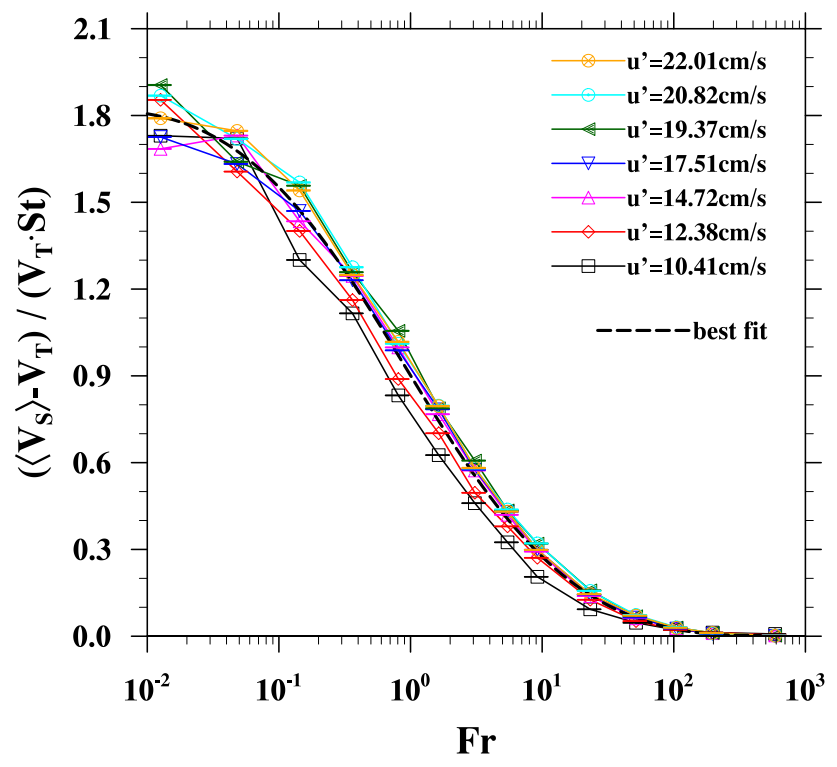

Figure 4: The difference between the average settling velocity and terminal velocity normalized by terminal velocity and Stokes number as a function of particle Froude number

one includes effects of $R_{\lambda}$. An important advantage of this decomposition is that the effects of inertia are separated from the effects of the flow Reynolds number. For the simulations discussed in this section, Eq. 12 reduces to

$$
\left\langle V_{S}\right\rangle-V_{T}=V_{T} \cdot S t \cdot \mathbf{F}_{4}(F r)
$$

By curve fitting we obtain the analytical formula of $\mathbf{F}_{4}$, namely

$$
\mathbf{F}_{4}(x)=\frac{p_{1} x^{2}+p_{2} x+p_{3}}{x^{2}+q_{1} x+q_{2}}
$$

where $x=\log _{10}(F r), p_{1}=0.8296, p_{2}=-4.184, p_{3}=5.316, q_{1}=0.2412$ and $q_{2}=5.897$.

The accuracy of the new parameterization is verified by comparing against DNS data. The comparison is presented in Fig. 5 We find a good quantitative agreement between the parameterization and DNS results for a wide range of the particle Froude number. In Section 3.3 we will extend this parameterization to different $R_{\lambda}$. 


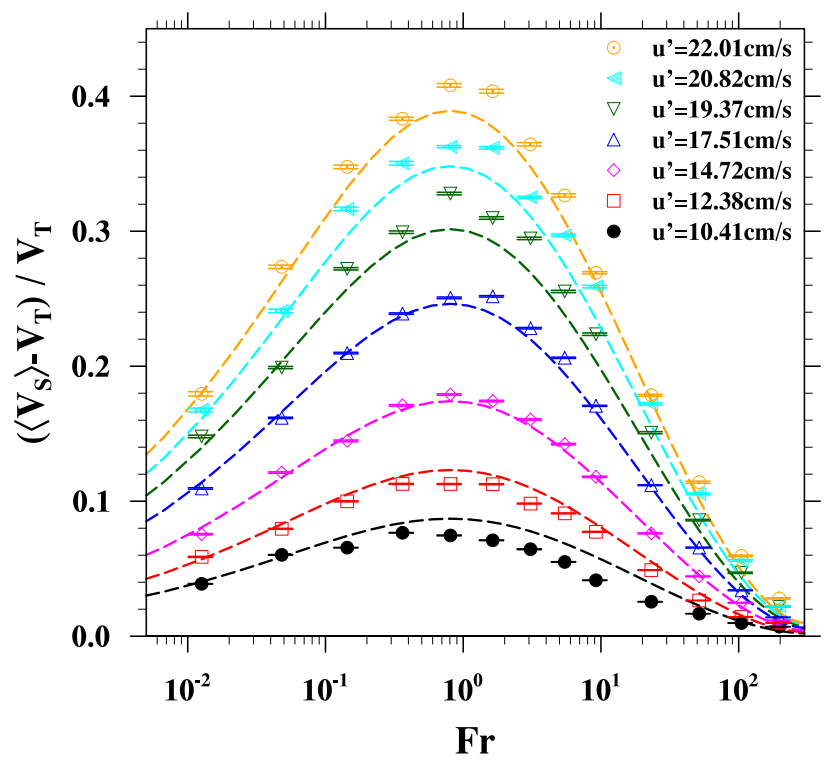

Figure 5: The difference between the average settling velocity and terminal velocity normalized by terminal velocity as a function of particle Froude number. Markers indicate results obtained from DNS. Dashed lines (with matching colors) refer to the new parameterization.

Dissipation of energy represents in part the intensity of local mixing by large scales in the turbulent flow. In isotropic homogeneous turbulence the average energy dissipation rate can be approximated using the large scale characteristics (Mouri et al., 2012), namely

$$
\langle\varepsilon\rangle \sim \frac{u^{\prime 3}}{L_{f}}
$$

where $L_{f}$ represents the size of the energy-containing eddies and can be set to the longitudinal integral length scale. Table 5 shows the mutual relation between the energy dissipation rate, the rms fluctuating velocity and $L_{f}$. The increase of $\varepsilon$ form 10 to $1000 \mathrm{~cm}^{2} / \mathrm{s}^{3}$ results in a threefold decrease of $L_{f}$ and a threefold increase of $u^{\prime}$. The increase of $u^{\prime}$ is the main factor affecting the settling velocity of inertial particles. Wang and Maxey (1993) showed that particles settling in the channel-like regions are indeed strongly affected by the velocity at large turbulent scales such as $u^{\prime}$. 
In this study we found that for a wide range of particle Froude numbers the relation between $u^{\prime 2}$ and $\left(\left\langle V_{S}\right\rangle-V_{T}\right)$ is linear (see Fig. 6). Since in all discussed DNS $u^{\prime} \propto \varepsilon^{1 / 4}$ the increase in the settling rate is also a linear function of $\sqrt{\varepsilon}$.

Table 5: Turbulence statistics (in physical units) of the simulated flows at $256^{3}$ using the stochastic forcing scheme and normalized droplet properties. The response time $\tau_{p}$ and terminal velocity $V_{T}$ correspond to droplets with a radius of $20 \mu \mathrm{m}$.

\begin{tabular}{rrrrrr}
\hline \hline$\varepsilon\left[\mathrm{cm}^{2} / \mathrm{s} 3\right]$ & $u^{\prime}[\mathrm{cm} / \mathrm{s}]$ & $L_{f}[\mathrm{~cm}]$ & $T_{e}[\mathrm{~s}]$ & $\tau_{p} / T_{e}$ & $V_{T} / u^{\prime} * 10^{2}$ \\
\hline 10 & 6.96 & 33.58 & 4.84 & 0.74 & 0.11 \\
50 & 10.41 & 22.46 & 2.17 & 0.49 & 0.24 \\
100 & 12.38 & 18.88 & 1.53 & 0.41 & 0.34 \\
200 & 14.72 & 15.88 & 1.08 & 0.35 & 0.48 \\
400 & 17.51 & 13.35 & 0.77 & 0.29 & 0.68 \\
600 & 19.37 & 12.07 & 0.63 & 0.26 & 0.83 \\
800 & 20.82 & 11.23 & 0.54 & 0.25 & 0.97 \\
1000 & 22.01 & 10.62 & 0.48 & 0.23 & 1.09 \\
\hline \hline
\end{tabular}

Interestingly, by changing the flow dissipation rate the relative importance of gravity vs particle inertia is modified. That is because both $S t$ and $S_{V}$ are functions of $\varepsilon$, namely $S t \propto \varepsilon^{1 / 2}$ and $S_{V} \propto \varepsilon^{-1 / 4}$. However, it does not imply that $\left(\left\langle V_{S}\right\rangle-V_{T}\right)$ is also a linear function of $S t$. The reason is that the Stokes number depends also on the particle size, namely $S t \propto a^{2}$.

An important question to pose here is whether the linear dependence on 380 $\sqrt{\varepsilon}$ is general or a characteristic only for this particular $R_{\lambda}$. To investigate the effect of Reynolds number, additional series of simulations at different $R_{\lambda}$ and different particle $\mathrm{Fr}$ have been performed. Results from these DNS are presented in Fig. 7 We confirm that linearity of the relationship does not depend on $R_{\lambda}$. However, the increase of the settling rate is larger at higher $R_{\lambda}$.

This issue will be analyzed more broadly in Section 3.3. 


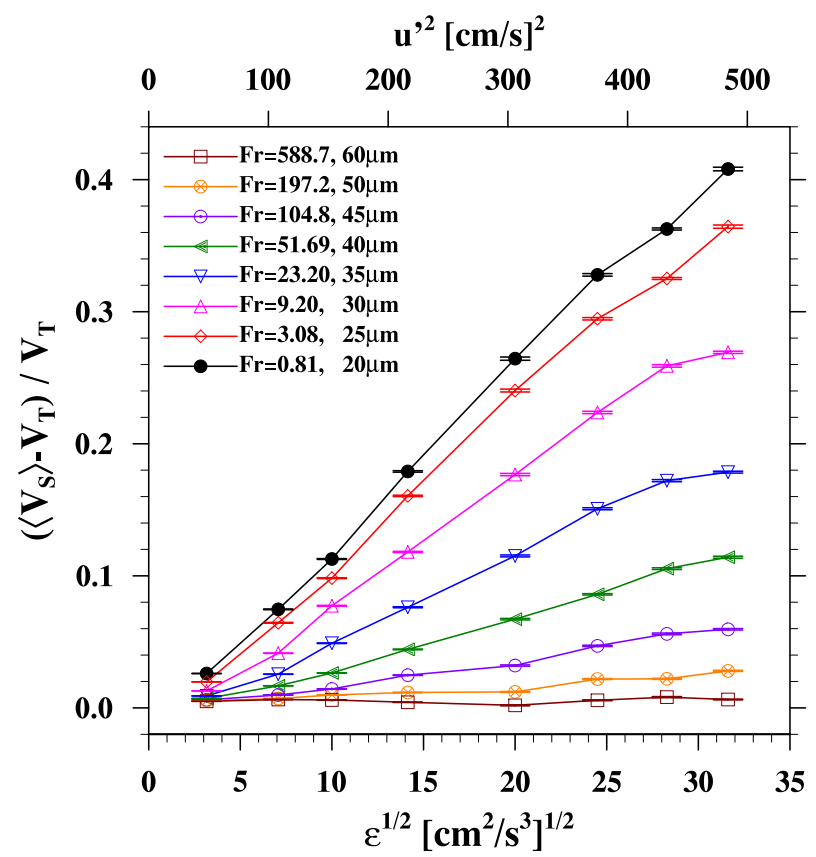

Figure 6: The difference between the average settling velocity and terminal velocity normalized by terminal velocity as a function of square root of energy dissipation rate and $u^{\prime 2}$. Different colors and markers correspond to different size of the inertial particles (cloud droplets).

\subsection{The effect of particle-to-fluid density ratio}

In the previous section we considered conditions which are characteristic for cloud processes in the atmosphere. In these simulations, we kept the density ratio fixed and equal to $\sigma=1000$. The results do not show a clear evidence of a reduction in the settling rate of small cloud droplets. In this section, we discuss the results of DNS performed at different density ratio in the range of $500<\sigma<10000$. To perform simulations at even lower density ratio (i.e. $\sigma<500$ ), other hydrodynamic forces (e.g., history term, added mass, etc.) will have to be included. The new set of simulations was performed at fixed energy dissipation rate $\varepsilon=800 \mathrm{~cm}^{2} / \mathrm{s}^{3}$. Other parameters such as gravity, fluid viscosity and density remain unchanged namely, $\mathbf{g}=9.8 \mathrm{~m} / \mathrm{s}^{2}, \nu=0.17 \mathrm{~cm}^{2} / \mathrm{s}$ and $\rho_{f}=0.001 \mathrm{~g} / \mathrm{cm}^{3}$.

Fig. 8 shows the increase $\left(\left\langle V_{S}\right\rangle-V_{T}\right)$ in the particle mean settling velocity 


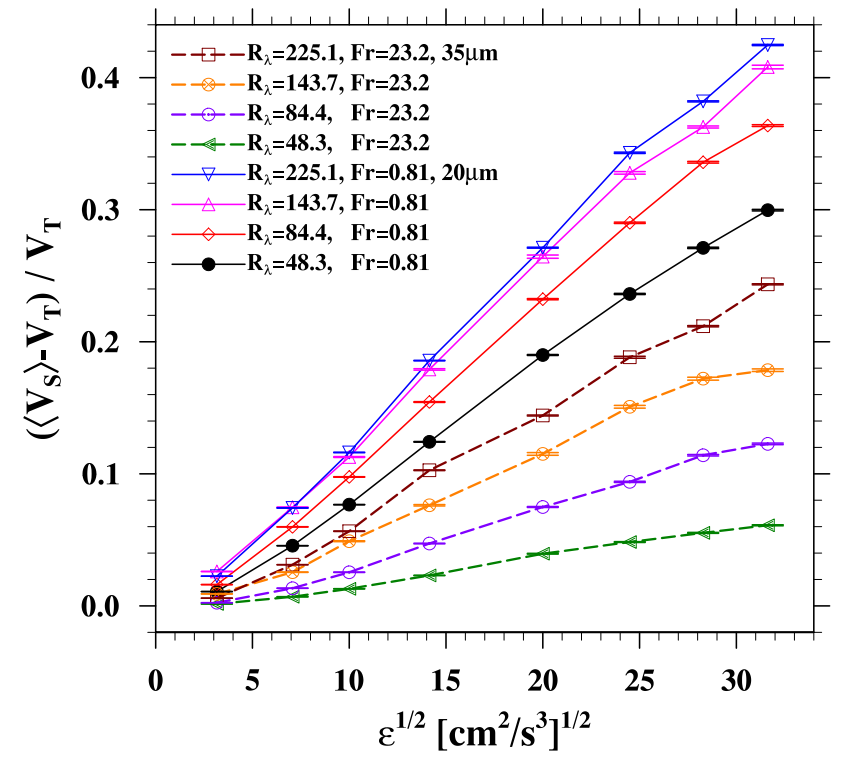

Figure 7: The difference between the average settling velocity and terminal velocity normalized by terminal velocity as a function of square root of energy dissipation rate. Simulations have been performed at four different $R_{\lambda}$ and droplets of size $a=20 \mu \mathrm{m}$ (solid lines) and $a=35 \mu \mathrm{m}$ (dashed lines).

normalized by the terminal velocity. Different colors correspond to different density ratio (with blue curve representing water droplets in turbulent air). Several conclusions emerge from Fig. 8 First, regardless of the density ratio, the average settling velocity is always larger or equal to the terminal velocity. Second, the maximum increase in the settling velocity does not exceed $36 \%$. This is a consequence of a finite and fixed value of the energy dissipation rate that is used in all simulations. Third, particles of high density (large $\sigma$ ) are less responsive to the turbulence eddies and therefore an increase in the settling velocity occurs only for particles with small radii (inertia).

The shape of the functions corresponding to different $\sigma$ is generally preserved. It is worth mentioning that the particle equation of motion can be rewritten in dimensionless form in terms of $S t$ and $S_{V}$. In our simulation the ratio $S t / S_{V}=\varepsilon^{3 / 4} /\left(g \nu^{1 / 4}\right)$ is constant and equal to 0.24 . Therefore, the results will collapse to a single curve when plotted as a nondimensional parameters $S t$ 
and $S_{V}$ (not shown for conciseness).



Figure 8: Increase in the particle mean settling velocity normalized by the terminal velocity as a function of particle radius. Different markers (colors) correspond to different density ratio ( $\sigma=1000$ - water droplets in air). Each simulation was performed assuming a fixed energy dissipation rate $\varepsilon=800 \mathrm{~cm}^{2} / \mathrm{s}^{3}$. Resolution of computational grid was $256^{3}$.

\subsection{The effect of flow Taylor microscale Reynolds number}

Another important parameter that affects the settling velocity of inertial particles is the range of turbulent scales present in the flow. Typically, the range of scales is defined by the Taylor microscale Reynolds number $\left(R_{\lambda}=u^{\prime} \lambda / \nu\right)$. In pseudo-spectral simulations $R_{\lambda}$ depends, among other things, on resolution of the computational grid, dealiasing truncation, forcing scheme and fluid viscosity. The DNS results discussed in previous section were limited to only one grid resolution $\left(256^{3}\right)$ and the Taylor microscale Reynolds number was fixed $\left(R_{\lambda}=\right.$ 143.74). To examine the effect of $R_{\lambda}$ on the settling rate, more simulations at various grid resolutions is needed. Performing DNS at large computational grids (e.g. $1024^{3}$ ) is numerically expensive and requires advanced and efficient codes 

computational cost was a major obstacle to address this problem in the past.

Our present DNS cover a wide range of flow Taylor microscale Reynolds numbers (up to 373 in simulations with stochastic forcing and up to 500 in simulations with deterministic forcing). The new simulations were performed at various grid resolutions $\left(32^{3}-1024^{3}\right)$. First, we analyze the settling velocity of low inertia particles relevant to small water droplets with 5 to $20 \mu \mathrm{m}$ radii. Other basic parameters are as follows: $\mathbf{g}=9.8 \mathrm{~m} / \mathrm{s}^{2}, \sigma=1000$ and $\nu=0.17 \mathrm{~cm}^{2} / \mathrm{s}$.

The results obtained in DNS are presented in Fig. 9a with markers. The increase of the settling velocity normalized by $V_{T}$ is plotted as a function of both $R_{\lambda}$ and grid size $(N)$. The energy dissipation rate is set to $\varepsilon=400 \mathrm{~cm}^{2} / \mathrm{s}^{3}$. The results indicate that the increase in the settling rate of low inertia particles ( $a=10 \mu \mathrm{m}, F r=0.013$ ) is insensitive to $R_{\lambda}$. An increase in the Reynolds number from 27.8 to 225 results only in $5 \%$ increase in the normalized settling velocity. The main observation concerns the saturation at large $R_{\lambda}$. This suggests that the largest eddies. For small $R_{\lambda}$ the settling velocity converges to the terminal velocity because the kinetic energy of small turbulent structures is not sufficient at this $R_{\lambda}$ to alter the particle settling velocity. For the particles of size $10<$ $a<20 \mu \mathrm{m}(0.013<F r<0.808)$ a saturation is still observed at a higher $R_{\lambda}$.

445 Additionally the increase in terminal velocity is larger, which is consistent with the results presented in Fig. 2.

Our results for larger particles $(25-60 \mu \mathrm{m}$ in radii, $1.6<F r<589)$ are shown in Fig. 9b. There are two essential differences between settling velocity of small and large particles. Regardless of the Reynolds number, the actual settling velocity tends to terminal velocity when the particle size increases. This is consistent with the conclusions from Fig. 2 and the results from large particle inertia $S t$ and large $S_{V}$.

By increasing the grid resolution, or equivalently the domain size (for a given value of $\varepsilon$ ), a larger turbulent scales are represented in the flow (see Table 6). 455

This in turn leads to an increase in the settling rate of the particles with larger 
Table 6: Basic characteristics of particles with respect to turbulent scales. Statistics are computed based on DNS data. The flow was forced by stochastic scheme. The energy dissipation rate was set to $\varepsilon=400 \mathrm{~cm}^{2} / \mathrm{s}^{3}$.

\begin{tabular}{|c|c|c|c|c|c|}
\hline$a[\mu \mathrm{m}]$ & 25 & 30 & 40 & 50 & 60 \\
\hline$S t$ & 0.40 & 0.57 & 1.01 & 1.58 & 2.28 \\
\hline \multirow[t]{2}{*}{$S_{V}$} & 2.79 & 4.01 & 7.14 & 11.15 & 16.06 \\
\hline & \multicolumn{5}{|c|}{$R_{\lambda}=27.7, N=32$} \\
\hline$\tau_{p} / T_{e}$ & 0.055 & 0.080 & 0.142 & 0.221 & 0.319 \\
\hline \multirow[t]{2}{*}{$V_{T} / u^{\prime}$} & 1.04 & 1.50 & 2.66 & 4.16 & 5.99 \\
\hline & \multicolumn{5}{|c|}{$R_{\lambda}=84.4, N=128$} \\
\hline$\tau_{p} / T_{e}$ & 0.017 & 0.025 & 0.045 & 0.070 & 0.101 \\
\hline \multirow[t]{2}{*}{$V_{T} / u^{\prime}$} & 0.59 & 0.86 & 1.52 & 2.39 & 3.43 \\
\hline & \multicolumn{5}{|c|}{$R_{\lambda}=373, N=1024$} \\
\hline$\tau_{p} / T_{e}$ & 0.004 & 0.006 & 0.010 & 0.016 & 0.023 \\
\hline$V_{T} / u^{\prime}$ & 0.28 & 0.41 & 0.72 & 1.13 & 1.64 \\
\hline
\end{tabular}

inertia. For example, the increase in the settling velocity of $60 \mu \mathrm{m}$ particles is noticeable only when $R_{\lambda}>150$. Yet, the increase is very small and does not exceed $2.4 \%$ at $R_{\lambda}=375$.

The DNS data are helpful to complete the parameterization proposed in Section 3.1. The present results show that the increase in the settling rate depends on $R_{\lambda}$ but this relationship is different for different Froude numbers. To separate the contribution of $\mathrm{Fr}$ (to $\left\langle V_{S}\right\rangle$ ) which results from different Reynolds numbers we need to find a function $m\left(R_{\lambda}\right)$ for which all data points in Fig. 9 (normalized by $S t$ and $\mathbf{F}_{4}$ ) will collapse to a single curve. Given $m=0$ at $R_{\lambda}=143.74$, we propose the following formula for the exponent $m$

$$
m\left(R_{\lambda}\right)=c_{1} \log _{10}\left(\frac{R_{\lambda}}{143.7}\right)+c_{2} \log _{10}\left(\frac{R_{\lambda}}{143.7}\right)^{2} .
$$

The coefficients $c_{1}=0.2125$ and $c_{2}=0.0175$ are determined using $2 \mathrm{D}$ least square optimization. 
The last step is to determine $\mathbf{F}_{5}$. We propose an analytical formula for $\mathbf{F}_{5}$ by fitting the second order polynomial to the discrete DNS data. The fitted function has the following form

$$
\mathbf{F}_{5}\left(\log _{10}\left(R_{\lambda}\right)\right)=-0.4005\left(\log _{10}\left(R_{\lambda}\right)\right)^{2}+2.4285 \log _{10}\left(R_{\lambda}\right)-2.338
$$

To verify the accuracy of the new parameterization we made a strict comparison of its values with the DNS data. In Fig. 9 the analytical predictions are plotted with dashed lines. The colors are consistent with the colors of markers used for plotting the DNS results. We confirm a good quantitative agreement between DNS and the parameterization for particles with low inertia $(F r<0.8)$. The analytical formula predicts a small reduction of $\left\langle V_{S}\right\rangle$ at large $R_{\lambda}$. For particles with medium $F r$ (i.e. $1<F r<20$ ) the parameterization yields a rather qualitative agreement. The trend at low $R_{\lambda}$ is reproduced but the saturation at large $R_{\lambda}$ is not correctly predicted. For particles with the Froude numbers $23.2<\mathrm{Fr}<51.7$ there is no saturation at large $R_{\lambda}$ and again we observe a good quantitative agreement between DNS and the parameterization. For particles with large Froude numbers $(F r>100)$ the increase in settling rate is very small (comparing to $V_{T}$ ). Such small differences are qualitatively reproduced by the parameterization.

The results presented in Fig. 9 are useful in designing future numerical simulations. For instance one can estimate the optimal size of the computational domain for a given particle size. Modeling of turbulence at resolution $1024^{3}$ or higher is numerically very expensive and in view of these results such effort may not be necessary.

\subsection{The effect of the large-scale forcing scheme}

Turbulent flows are dissipative, therefore to maintain their stationarity a continuous supply of energy is required. In direct numerical simulations the turbulent flow is driven by an external forcing to avoid turbulence decay. In pseudospectral methods the kinetic energy is injected in the Fourier space to lowwavenumber modes. Restricting the forcing to large scales only is thought to en- 
(a)



(b)

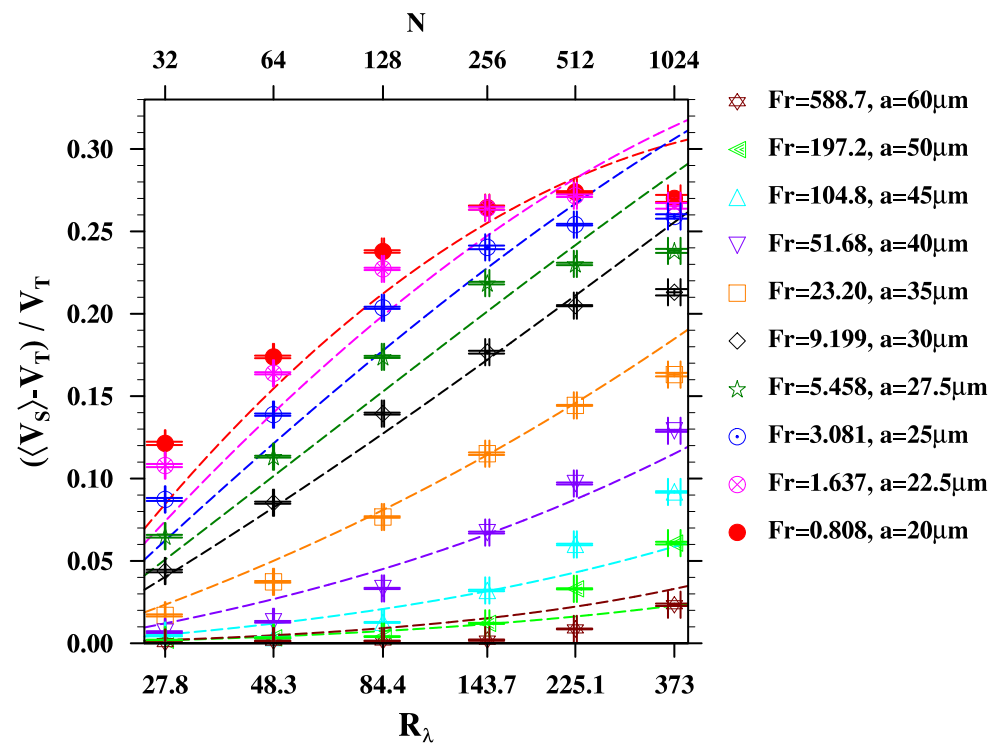

Figure 9: The increase in average settling velocity normalized by $V_{T}$ as a function of Taylor microscale Reynolds number and size of the computational grid. Markers indicate results obtained from DNS. Dashed lines (with matching colors) refer to the parameterization. (a) Results for particle radii in the range 10-20 $\mu \mathrm{m}$. The increase in settling velocity grows with $F r$. (b) Results for particle radii in the range 20-60 $\mu \mathrm{m}$. For this range, unlike (a), the increase in settling velocity decreases with $\mathrm{Fr}$. 
sure that the small scales of the flow are unaffected by the forcing (Ishihara et al., 2009).

The simulations analyzed in previous sections have been performed using the stochastic forcing (Eswaran and Pope, 1988; Rosa et al., 2015) which is based on six Uhlenbeck-Ornstein random processes (Eswaran and Pope, 1988). The energy is continuously injected to the 80 low-wavenumber modes, namely $(|\mathbf{k}|<$ $\sqrt{8})$.

Since the forcing scheme affects the large scales (energetic scales) of the flow, it raises a question about sensitivity of the DNS results to the forcing method. In this section we address this question by comparing two sets of simulations performed with two different forcing methods. The supplementary set of simulations was performed with deterministic forcing (Sullivan et al., 1994). In deterministic forcing the energy levels of the two lowest wavenumber shells $(0.5<|\mathbf{k}|<1.5$ and $1.5<|\mathbf{k}|<2.5)$ are fixed to $E(1)=0.55544$ and $E(2)=0.159843$, respectively.

Fig. 10 shows the average settling velocity of inertial particles in a turbulent flow normalized by the terminal velocity as a function of $\ln (F r)$, particle radius, $\ln \left(R_{\lambda}\right)$ and normalized turbulent intensity. The simulations were performed assuming $\mathbf{g}=9.8 \mathrm{~m} / \mathrm{s}^{2}, \nu=0.17 \mathrm{~cm}^{2} / \mathrm{s}, \sigma=1000$ and $\varepsilon=400 \mathrm{~cm}^{2} / \mathrm{s}^{3}$. Fig. 10. 515 compares the results obtained with two different forcing schemes namely, (a) stochastic and (b) deterministic. Both methods show a similar trend, although there is a significant difference in the velocity magnitude for a given particle size and mesh resolution $(N)$. At fixed resolution, particles settle faster in the flow forced by the deterministic scheme. This partially results from the different flow Reynolds numbers. Namely, the flow statistics show that the deterministic scheme leads to a higher flow Reynolds number. This in turn can be related to larger size of the energetic eddies which are responsible for particle acceleration. It should be noted that the results are consistent with the results presented in Fig. 9] where larger $R_{\lambda}$ leads to larger $\left\langle V_{S}\right\rangle$.

To facilitate a quantitative comparison of the results obtained with different forcing schemes, the data presented in Fig. 10 are tabulated in Tables 7 and 8 . 
(a)

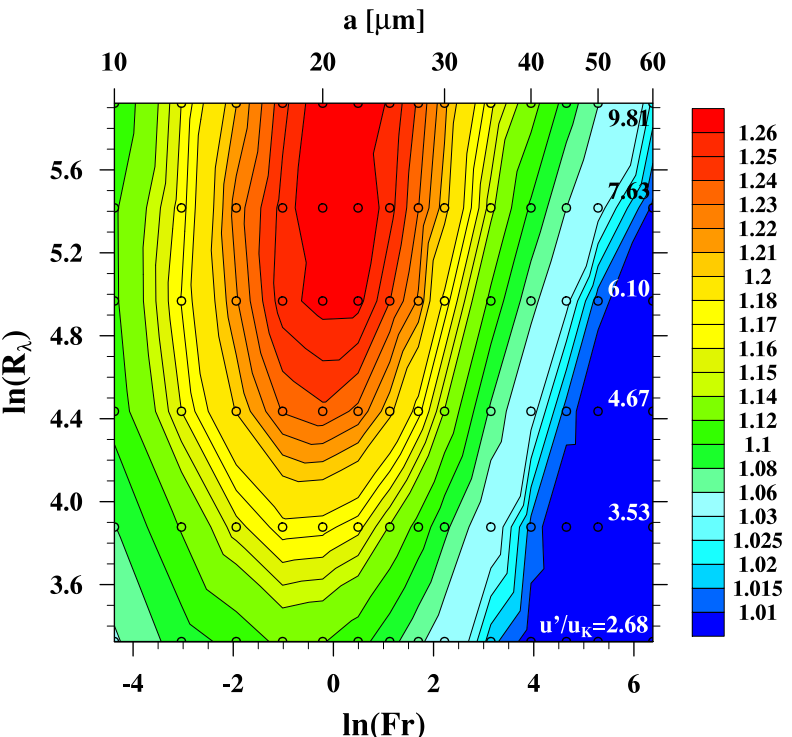

(b)



Figure 10: The average settling velocity of inertial particles relevant to cloud droplets in turbulent air normalized by the terminal velocity as a function of $\ln (F r)$, particle radius, $\ln \left(R_{\lambda}\right)$ and normalized turbulent intensity. DNS results obtained with (a) stochastic forcing, (b) deterministic forcing. 
Table 7: The average settling velocity of cloud droplets in turbulent flow. The simulations were performed at six different grid resolutions using stochastic forcing scheme. Numerical uncertainties do not exceed $0.1 \%$.

\begin{tabular}{|c|c|c|c|c|c|c|c|}
\hline & $N=$ & 32 & 64 & 128 & 256 & 512 & 1024 \\
\hline & $R_{\lambda}=$ & 28 & 48 & 84 & 144 & 225 & 372 \\
\hline$a$ & $V_{T}[\mathrm{~cm} / \mathrm{s}]$ & \multicolumn{6}{|c|}{$\left\langle V_{S}\right\rangle[\mathrm{cm} / \mathrm{s}]$} \\
\hline 10 & 1.281 & 1.354 & 1.382 & 1.420 & 1.430 & 1.431 & 1.411 \\
\hline 12.5 & 2.001 & 2.182 & 2.245 & 2.322 & 2.343 & 2.332 & 2.322 \\
\hline 15 & 2.882 & 3.192 & 3.331 & 3.461 & 3.513 & 3.530 & 3.486 \\
\hline 17.5 & 3.923 & 4.414 & 4.612 & 4.829 & 4.928 & 4.918 & 4.890 \\
\hline 20 & 5.124 & 5.746 & 6.015 & 6.343 & 6.479 & 6.528 & 6.508 \\
\hline 22.5 & 6.485 & 7.185 & 7.548 & 7.959 & 8.198 & 8.247 & 8.207 \\
\hline 25 & 8.006 & 8.706 & 9.118 & 9.637 & 9.930 & 10.041 & 10.081 \\
\hline 27.5 & 9.688 & 10.318 & 10.785 & 11.372 & 11.806 & 11.919 & 11.995 \\
\hline 30 & 11.529 & 12.035 & 12.513 & 13.137 & 13.566 & 13.892 & 13.986 \\
\hline 35 & 15.693 & 15.959 & 16.278 & 16.896 & 17.499 & 17.959 & 18.252 \\
\hline 40 & 20.497 & 20.636 & 20.765 & 21.179 & 21.874 & 22.485 & 23.140 \\
\hline 45 & 25.941 & 26.061 & 26.031 & 26.270 & 26.773 & 27.498 & 28.324 \\
\hline 50 & 32.026 & 32.086 & 32.132 & 32.157 & 32.414 & 33.085 & 33.974 \\
\hline 60 & 46.118 & 46.137 & 46.180 & 46.184 & 46.207 & 46.521 & 47.197 \\
\hline
\end{tabular}

\subsection{The effect of gravity}

Next, we extend the results beyond cloud droplets, allowing particle Stokes number and dimensionless sedimentation velocity $S_{V}$ to vary independently. We note that the effects of gravity on particle acceleration and relative velocity in the independent $S t-S_{V}$ space is recently studied in (Parishani et al., 2015). Hence, here we extend and explore the results for different values of gravity to allow a varying $S_{V}$ while keeping the Stokes number constant. Eight series of simulations were performed for a fixed set of parameters, namely, $u^{\prime}=20.82 \mathrm{~cm} / \mathrm{s}$, 
Table 8: As in Table 7 but for deterministic forcing.

\begin{tabular}{|c|c|c|c|c|c|c|c|}
\hline & $N=$ & 32 & 64 & 128 & 256 & 512 & 1024 \\
\hline & $R_{\lambda}=$ & 44 & 77 & 121 & 197 & 303 & 499 \\
\hline$a$ & $V_{T}[\mathrm{~cm} / \mathrm{s}]$ & \multicolumn{6}{|c|}{$\left\langle V_{S}\right\rangle[\mathrm{cm} / \mathrm{s}]$} \\
\hline 10 & 1.281 & 1.420 & 1.435 & 1.433 & 1.440 & 1.453 & 1.423 \\
\hline 12.5 & 2.001 & 2.283 & 2.353 & 2.355 & 2.355 & 2.386 & 2.399 \\
\hline 15 & 2.882 & 3.470 & 3.544 & 3.564 & 3.581 & 3.611 & 3.540 \\
\hline 17.5 & 3.923 & 4.768 & 4.949 & 4.970 & 5.005 & 5.079 & 5.075 \\
\hline 20 & 5.124 & 6.303 & 6.490 & 6.674 & 6.695 & 6.684 & 6.677 \\
\hline 22.5 & 6.485 & 7.941 & 8.182 & 8.355 & 8.423 & 8.501 & 8.501 \\
\hline 25 & 8.006 & 9.706 & 9.949 & 10.238 & 10.366 & 10.373 & 10.417 \\
\hline 27.5 & 9.688 & 11.468 & 11.827 & 12.104 & 12.237 & 12.417 & 12.496 \\
\hline 30 & 11.529 & 13.296 & 13.802 & 14.118 & 14.248 & 14.481 & 14.745 \\
\hline 35 & 15.693 & 17.435 & 17.901 & 18.361 & 18.545 & 18.880 & 19.311 \\
\hline 40 & 20.497 & 22.023 & 22.423 & 22.851 & 23.567 & 23.705 & 24.097 \\
\hline 45 & 25.941 & 27.057 & 27.533 & 28.003 & 28.901 & 29.244 & 29.375 \\
\hline 50 & 32.026 & 32.665 & 33.757 & 34.200 & 34.774 & 34.812 & 35.663 \\
\hline 60 & 46.118 & 45.922 & 47.001 & 47.675 & 48.477 & 48.907 & 49.852 \\
\hline
\end{tabular}

$535 \varepsilon=800 \mathrm{~cm}^{2} / \mathrm{s}^{3}, \nu=0.17 \mathrm{~cm}^{2} / \mathrm{s}$ and $\rho_{p} / \rho_{f}=1000$. In each series the Stokes number is determined by particle size. The range of considered Stokes numbers is $0.0224-1.815 . S_{V}$ is a function of gravity and depending on particle inertia varies from 0.094 to 45.58 . We assumed that the particle density corresponds to that of water $\left(\rho_{p}=1 \mathrm{~g} / \mathrm{cm}^{3}\right)$ while the fluid density corresponds to that of air ${ }_{540}\left(\rho_{f}=0.001 \mathrm{~g} / \mathrm{cm}^{3}\right)$.

There are two limiting cases for this problem. First, for $S_{V}=0$ (corresponding to $\mathbf{g}=0$ ) and the second when $S_{V}$ is large (equivalently $\mathbf{g} \rightarrow \infty$ ). In both cases, the particle velocity should be equal to the terminal velocity. Specifically, when $\mathbf{g}=0$, there is no featured direction and therefore both average particle 
velocity and terminal velocity are zero. For a large $S_{V}$, turbulence does not play a significant role and therefore the particle settling velocity should be equal to the terminal velocity. Nontrivial effect of gravity on the settling velocity of particles can be observed for intermediate values of $S t$ and $S_{V}$.

Fig. 11] shows the ratio of the average settling velocity of particles to the terminal velocity as a function of $S_{V}$. Each curve corresponds to a series of simulations with a fixed Stokes number. The settling velocity of particles with higher inertia $S t>0.2$ quickly converges to the terminal velocity (and hence a large slope) when gravity increases. This is the consequence of reduction in particle-eddy interaction time as particles settle faster at higher g. For low inertia particles $(S t=0.0223$ and 0.0504$)$, however, we observe a slight increase in the settling velocity when gravity increases from $1 \mathrm{~g}$ to $2 \mathbf{g}$ (the first two data points of each set). This increase may be due to larger $F r$ number $\left(F r \propto \mathbf{g}^{2}\right)$. On the other hand, in the limit $S_{V} \rightarrow 0$, low inertia particles behave like fluid elements and consequently $\left\langle V_{S}\right\rangle \rightarrow V_{T}$. For larger values of $S_{V}$ the actual settling velocity gradually converges to the terminal velocity.

\subsection{The effect of drag nonlinearity}

In the introduction we presented different and sometimes contradictory conclusions resulting from the previous studies in which a nonlinear drag was considered. Therefore those results may not be reconciled quantitatively as for the effect of nonlinear drag on the settling velocity. In the present study we address this problem by comparing the different formulations of the drag coefficients and number of simulations with different turbulence intensity and particle inertia (size).

The most common representations of the drag coefficient are: the Stokes drag, the standard nonlinear drag (Clift et al., 1978; Stout et al., 1995) and the square drag (Fung, 1993). We should point out that the square drag is unphysical for small particle Reynolds numbers. The mathematical formulas of these coefficients are given in Table 9. The two representations of the nonlinear drag show that $f$ is a function of the particle Reynolds number only. The square 


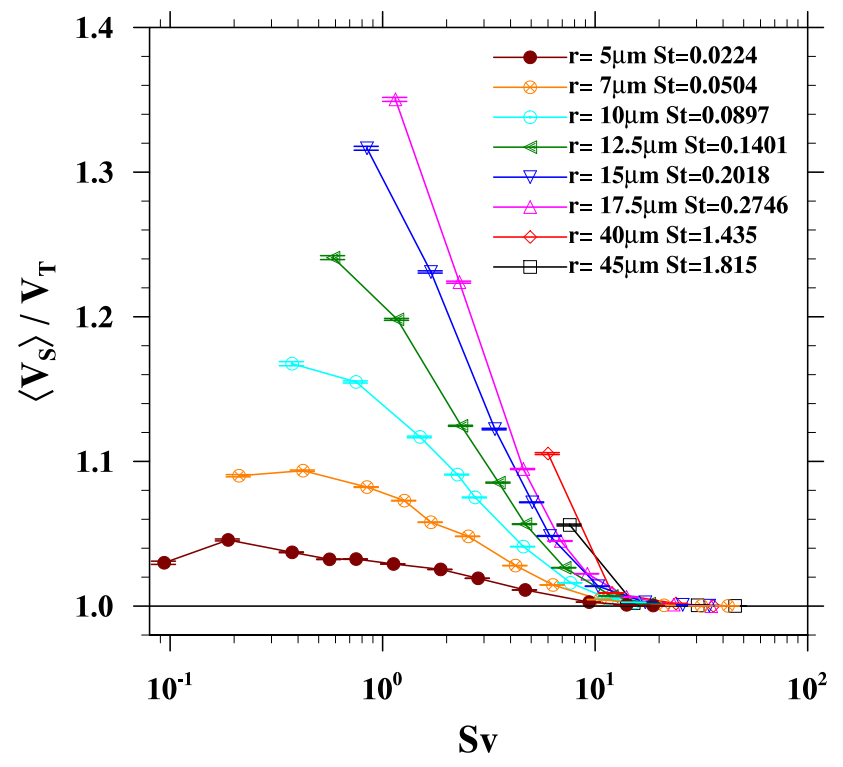

Figure 11: The ratio of the average settling velocity of particles to the terminal velocity as a function of $S_{V}$. The particles at each set have a fixed Stokes number as in the legend. Different colors correspond to different $S t$. The parameter $S_{V}$ was adjusted by varying the gravitational acceleration. The simulations were performed at grid resolution of $256^{3}$ assuming $\varepsilon=800 \mathrm{~cm}^{2} / \mathrm{s}^{3}$.

drag depends additionally on the density ratio and the Froude number. For a more extended discussion of the drag factor, including turbulence effects, compressibility and different flow regimes we refer to Crowe et al. (2011) Chapter 4.3.1.

Fig. 12 shows a quantitative comparison of the drag coefficients computed using four different formulations as a function of the particle Reynolds number, density ratio and $F r$. Interestingly, for the considered range of $R e$, there is only a slight difference between the Stokes drag and those of Clift et al. (1978) and Stout et al. (1995). However, the difference between the square drag and the three other formulations can be large, especially for small Fr. To predict the impact of the square drag on the settling velocity we should consider two extreme cases. First, when the particle has large inertia the drag coefficient goes to zero, so the settling velocity should be comparable with terminal velocity. Second, 
Table 9: Different formulation of drag factor

\begin{tabular}{|c|c|}
\hline Stokes drag & $f=1$ \\
\hline Nonlinear drag (Clift et al., 1978) & $f=1+0.15 R e^{0.687}$ \\
\hline Nonlinear drag (Stout et al., 1995$)$ & $f=\frac{C_{D}}{24} \operatorname{Re}$ where $C_{D}=\frac{1}{4}+\frac{24}{R e}+\frac{6}{\sqrt{R e}}$ \\
\hline Square drag (Fung, 1993) & $f=\frac{|\mathbf{U}-\mathbf{V}|}{V_{T}}=R e \sqrt{\frac{\sigma}{18 F r}}$ \\
\hline
\end{tabular}

when particle has very low inertia the drag force acting is very large. This makes the equation of motion unstable, and it is necessary to use a very short time step for integration of the equation of motion. After a relaxation period the droplets will behave as a fluid elements due to the strong drag force.



Figure 12: Drag coefficients as a function of particle Reynolds number $R e=|\mathbf{U}-\mathbf{V}| d / \nu$. Different colors correspond to different formulations. For square drag we assumed $\sigma=1000$.

Fig. 13 shows the mean particle settling velocity computed in simulations with both Stokes drag and nonlinear drag (Clift et al., 1978). All simulations have been performed at $256^{3}$ grid resolution with turbulent Reynolds num- 
Wang and Maxey (1993) and we conclude that the differences in the average settling velocity computed with the different drag forces are relatively small. The differences do not grow with the turbulence intensity. We confirm that the nonlinear drag (Clift et al., 1978) only slightly reduces the net increase in the

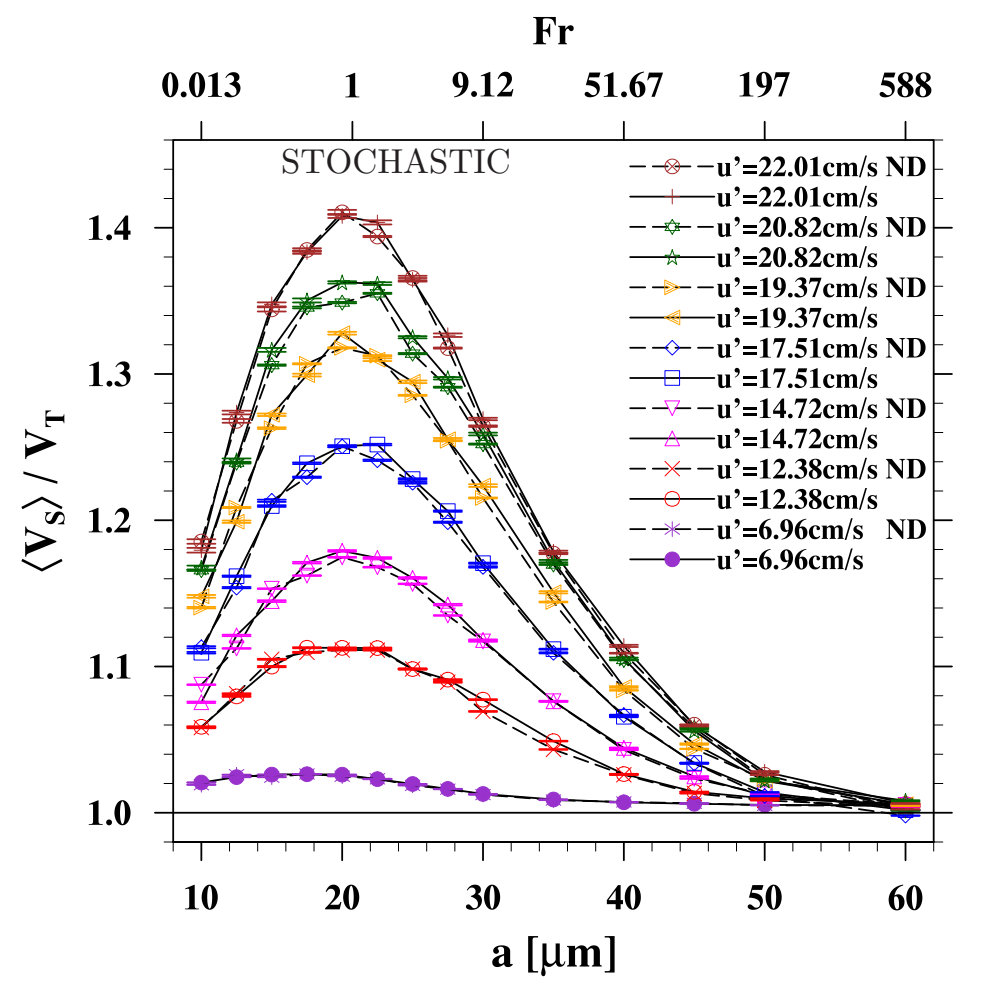

Figure 13: The mean settling velocity normalized by terminal velocity (computed with the Stokes drag) as a function of droplet radii. Different colors correspond to different turbulence intensity. Solid lines represent results computed with the Stokes drag. Results computed with nonlinear drag (Clift et al., 1978) are shown by dashed lines. The simulations were performed at grid resolution of $256^{3}$.

The next step is to quantify the impact of the square drag on the settling rate. The large differences between drag coefficients presented in Fig. 12 may suggest that the effect of the square drag on the settling rate can be greater 
than those presented in Fig. 13. In Fig. 14 we compare the mean particle settling velocity (normalized by terminal velocity characteristic for the Stokes drag) computed using three different formulation of the drag force. All series of simulations have been performed at $R_{\lambda}=143.74$ and energy dissipation rate of $\varepsilon=400 \mathrm{~cm}^{2} / \mathrm{s}^{3}$. We conclude that the difference in the settling rate computed using three different formulations of the drag force is small and does not exceed 1\%. The most visible difference is observed between the Stokes drag and the square drag using particle (water droplets) of $a \approx 20 \mu \mathrm{m}$ in radii.

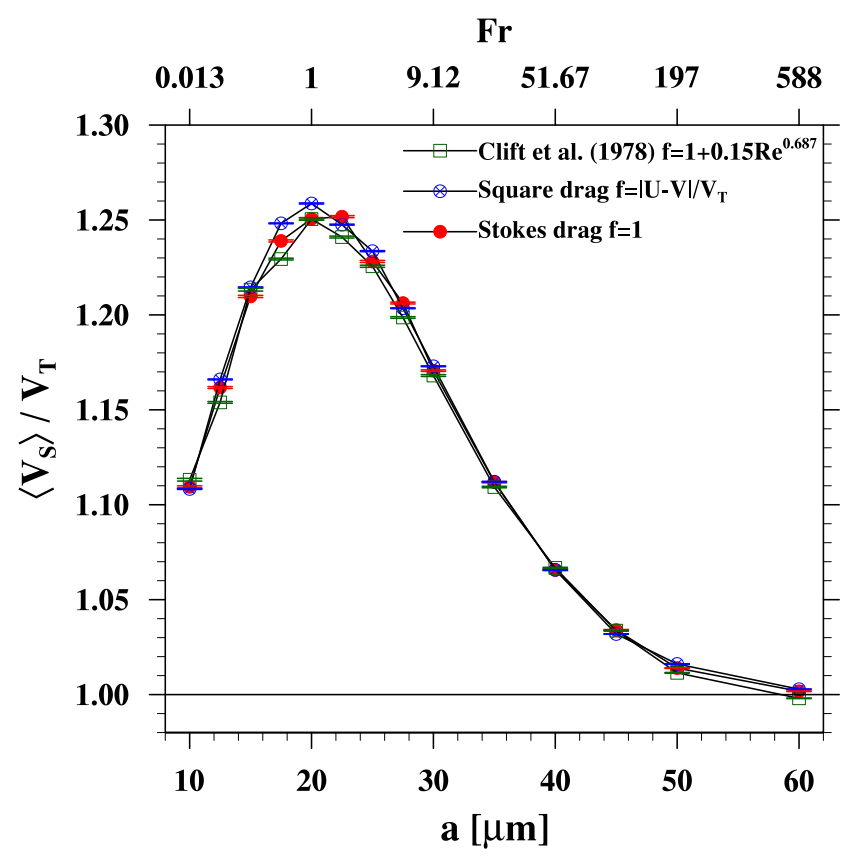

Figure 14: Effect of the drag force on the settling rate. Different colors correspond to simulations with different drag coefficients.

The presented results do not show a significant reduction in the settling velocity if the particles are subjected to the nonlinear drag. For large particles $(60 \mu \mathrm{m})$ the visible differences (see Fig. 13) between simulations performed with 615 Stokes and nonlinear drags are of order of the statistical uncertainty. In this regards, our results are different from those presented by Good et al. (2014). They showed that when the nonlinear drag is considered, the settling velocity 
of heavy inertial particles $\left(S_{V_{l}}=\tau_{p} g / u^{\prime}>1\right)$ is smaller than the corresponding terminal velocity. The reduction is up to $\left(V-V_{T}\right) / u^{\prime} \approx-0.1$. Interestingly, they obtained a similar trend both in DNS and in experiment. It is worth noting that the range of particle Reynolds number covered in their DNS (Fig. 3a therein) is approximately two times wider than in the present study. The main difference between the present work and Good et al. (2014) simulations lies in the flow forcing method. All our simulations with the nonlinear drag were performed using the stochastic forcing (Eswaran and Pope, 1988; Rosa et al., 2015) while Good et al. (2014) performed simulations using a deterministic forcing. In order to make a strict comparison with Good et al. (2014) study, we perform an additional set of simulations with the deterministic forcing scheme. Results from the later simulations are shown in Fig 15. Three series of simulations correspond ${ }_{630}$ to three values of energy dissipation rate, namely 100, 400 and $800 \mathrm{~cm}^{2} / \mathrm{s}^{3}$. Additionally we considered a wider range of particle radii i.e. 10-100 $\mu \mathrm{m}$.

DNS results computed using deterministic forcing are consistent with those obtained with the stochastic forcing. We do not observe a clear evidence of reduction in the settling velocity of large inertial particles when the nonlinear drag is used. It is worth noting that we run the simulations at least $100 T_{e}$. The statistics were collected after $40 T_{e}$ and averaged over at least $60 T_{e}$. Good et al. (2014) averaged their statistics over about $10 T_{e}$. The initial flow stabilization time and also the total time over which the averaging is performed may play a critical role in understanding the difference between our results and those presented by Good et al. (2014). Rosa et al. (2015) showed that the settling mechanism depends on particle inertia and $R_{\lambda}$. Large-inertia particles accumulate in the downward flow regions forming elongated (filament-like) structures. The elongated structures are stable and remain for a long time because the gravitational settling dominates the mechanism of turbulent mixing. We anticipate 645 that for such elongated structures the different types of drag force, namely the nonlinear versus Stokes drag, may not have a significant impact on the settling velocity. 


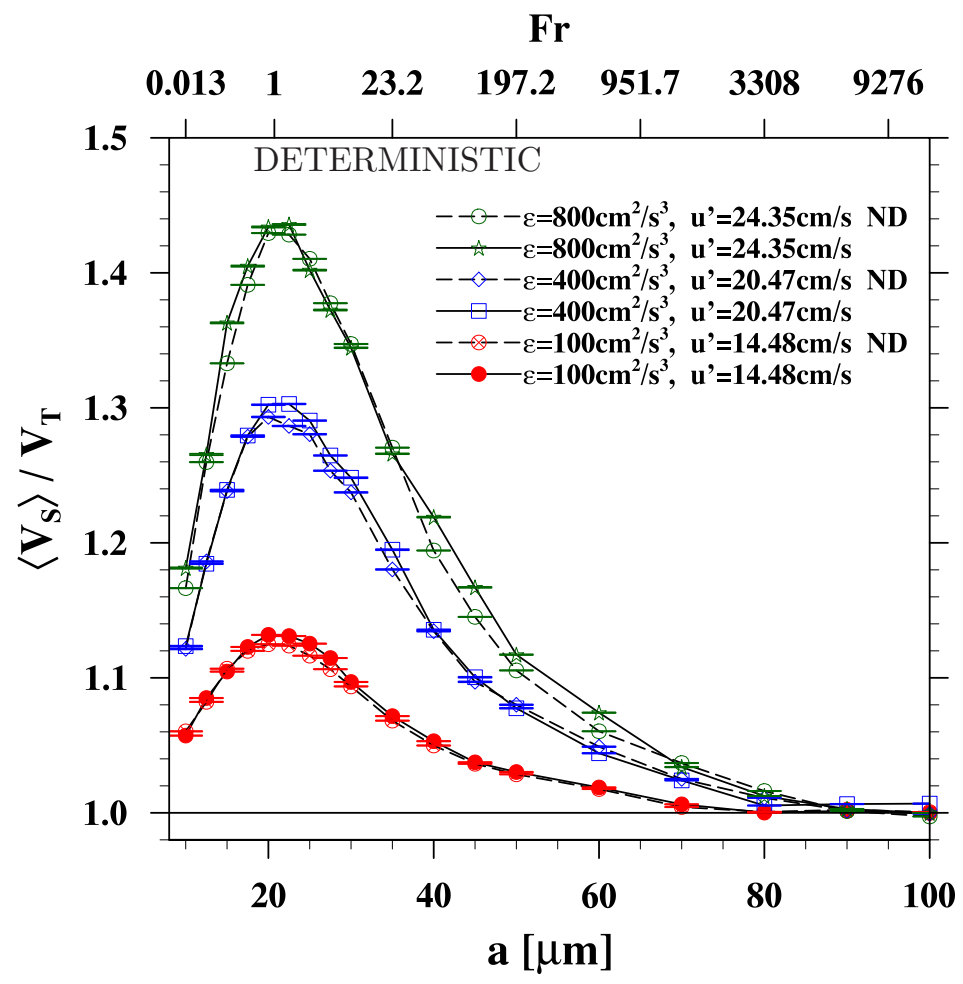

Figure 15: As in Fig 13 but for deterministic forcing. All series of simulations have been performed at the same grid size i.e. $256^{3}$ and turbulent Reynolds number $R_{\lambda}=196.87$.

\section{Effect of forced loitering}

The DNS results presented in the previous sections show that the turbulent flow increases the average settling velocity of the inertial particles. This increase is due to preferential sweeping (Wang and Maxey, 1993) which dominates other mechanisms that may slow down the settling rate, such as vortex trapping or loitering. An examination of the relative importance of the different mechanisms in a three-dimensional unsteady flow with a large number of particles is challenging. Recently, Good et al. (2014) performed numerical simulations in which the two mechanisms (i.e. preferential sweeping and loitering) were separated. This was done through blocking the particle motion in the horizontal direction, i.e. in direction perpendicular to the gravity. Blocking of 
the horizontal motion prevents natural tendency of the particles to sweep in the

downward flow regions and therefore restricts the particle-eddy interaction only in the vertical direction. They observed a reduced settling velocity of inertial particles in such conditions. In the present study we follow this idea. Four series of DNS simulations have been performed at four different values of turbulence intensity. Fig. 16] shows the difference between the average settling velocity and the associated terminal velocity (computed using the Stokes drag) for a given particle size. The different lines correspond to different values of energy dissi-

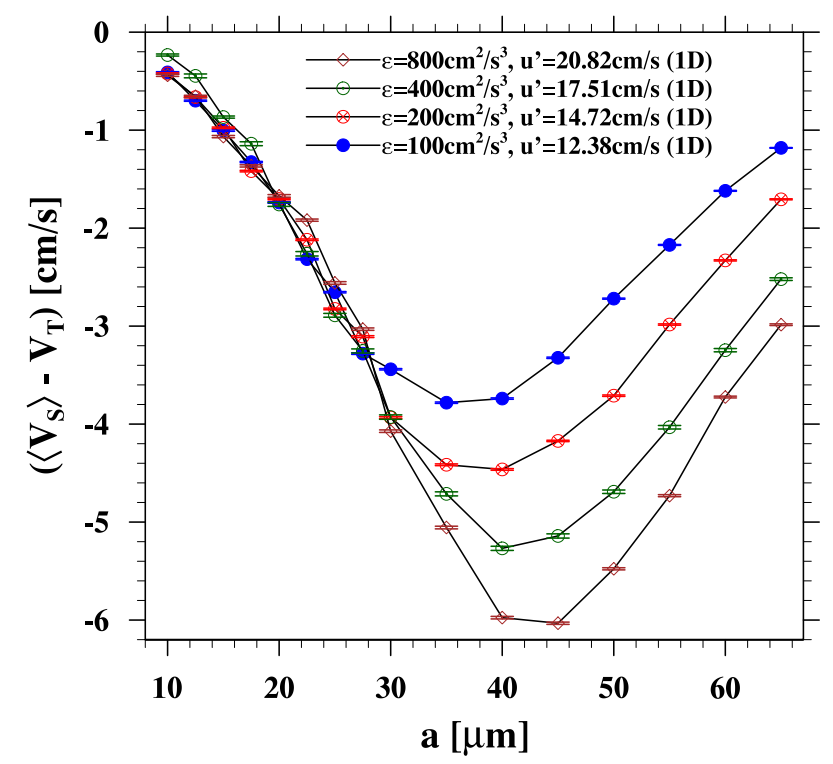

Figure 16: Effect of the blocked lateral motion on the settling rate. The simulations have been performed excluding particle motion in the horizontal direction (perpendicular to gravity).

pation rate. Several conclusions can be drawn from Fig. 16. First, the average settling velocity is lower than the terminal velocity for all particle/flow settings. This reduction is expected as the mechanism of preferential sweeping is disabled. Second for the droplets with radii smaller than $27.5 \mu \mathrm{m}$ the reduction is insensitive to energy dissipation rate. Third, the maximal reduction of the settling velocity takes place for droplets with radii $\approx 40 \mu \mathrm{m}$. Presence of this minimum 
indicates that for large particles the sampling of $U$ along the trajectory may have a dominant effect, as explained next. Rewriting particle equation of motion as $\langle V\rangle-\tau_{p} g=\langle U\rangle-\tau_{p}\langle d V / d t\rangle$ and noting that the acceleration term on the right hand side is zero, it is evident that the reduction in particle sedimentation velocity depends strongly on selective behavior of $\langle U\rangle$ at particle location. Namely, small particles $(a<27.5 \mu \mathrm{m})$ experience a similar $\langle U\rangle$ dynamics irrespective of the energy dissipation rate while larger particles sample a different fluid velocity on their trajectory causing the particle sedimentation response to change with $\varepsilon$.

\section{Conclusions}

Using direct numerical simulations, we have examined the different mechanisms and factors affecting the average settling velocity of inertial particles in a turbulent flow. The main focus is on water droplets of $10-60 \mu \mathrm{m}$ in radius suspended in air. It has been shown that the difference between the particle settling velocity and their terminal velocity is a roughly linear function of $\sqrt{\varepsilon}$. Simulations with the Stokes drag and various density ratios show that the average settling velocity is consistently larger or equal to the terminal velocity. This conclusion is in agreement with the recent study of Good et al. (2014). Additionally, we showed that the maximum increase in the settling velocity highly depends on the turbulence intensity or the energy dissipation rate at fixed $R_{\lambda}$ and kinematic viscosity.

Furthermore, effects of the Taylor microscale Reynolds number on the settling rate have been investigated. Consistent with the observations of Yang and Lei (1998), we conclude that at a fixed energy dissipation rate and a low Taylor microscale Reynolds number $\left(R_{\lambda}<100\right)$ the maximum level of increase scales with $R_{\lambda}$ and hence $u^{\prime}$. On the other hand, for larger $R_{\lambda}$ the increase in settling velocity saturates and this saturation is observed only for low-inertia particles $(a<30 \mu \mathrm{m})$.

The DNS results were used to develop an empirical parameterization that 
relates the settling velocity to three nondimensional parameters, namely, $S t, F r$ and $R_{\lambda}$. The accuracy of the new parameterization has been verified by comparing against DNS data. For a wide range of the nondimensional parameters we obtained quantitative agreement between the DNS results and the parameterization. Only for large $R_{\lambda}$ and $1<F r<20$ the parameterization yields a rather qualitative agreement.

In order to examine the possible effects of the forcing mechanism, our simulations were carried out using two different forcing schemes. Results obtained using both stochastic and deterministic forcing method show a similar trend, although there is a significant difference in magnitude of the settling velocity for a given particle radius and $R_{\lambda}$. At a given grid resolution, particles settle faster in the flow forced by the deterministic scheme. This is partially caused by the different values of $R_{\lambda}$. Flow statistics show that the deterministic scheme allows a higher $R_{\lambda}$ to be obtained. We expect the deterministic scheme to yield more coherent turbulent eddies. On the other hand the random nature of the stochastic forcing may affect the preferential concentration and consequently the settling velocity.

Additionally, we have examined the role of the gravitational acceleration. It has been shown that a larger gravity magnitude pushes the settling velocity of high-inertia particles $(S t>0.2)$ to quickly converge to their terminal velocity. For low-inertia particles $(S t<0.0504)$, however, we observe a small increase in the settling velocity when gravity is increased from $1 \mathrm{~g}$ to $2 \mathrm{~g}$.

Another important issue explored in our study is the non-linear drag and its effect on particle settling rate. In the considered range of Stokes number and $S_{V}$, the difference in the settling velocity due to different drag forces is very small and is independent of the energy dissipation rate. Our results are in qualitative agreement with the results of Wang and Maxey (1993). We confirm that the nonlinear drag slightly reduces the net increase in the settling velocity.

Finally, we performed simulations in which the horizontal motion of particles is blocked. Preventing horizontal motion turns off the preferential sweeping mechanism, which has a significant impact on the settling velocity of particles. 
Turning the preferential sweeping off causes the average settling velocity to be lower than the terminal velocity. Consistent with the recent study of Good et al. 2014) and Parishani et al. (2015) this implies that the dynamics of particleeddy interaction in the horizontal plane plays a central role in influencing the particle velocity in the vertical direction.

\section{Acknowledgements}

The authors would like to express sincere gratitude to Interdisciplinary Centre for Mathematical and Computational Modeling (ICM) at Warsaw University for providing computational resources (grant G49-15). This work was partially supported by the US National Science Foundation (NSF) under grants AGS-1139743 and CBET-1235974. Computing resources are provided by National Center for Atmospheric Research through CISL-P35751014 and CISLUDEL0001.

\section{References}

Afonso, M.M., 2008. The terminal velocity of sedimenting particles in a flowing fluid. J. Phys. A: Math. Theor. 41. doi:10.1088/1751-8113/41/38/385501

Alipchenkov, V.M., Zaichik, L., 2009. Effect of particle clustering on the gravitational settling velocity in homogeneous turbulence. Fluid Dynamics 44, 397-404. doi:10.1134/S0015462809030077.

Aliseda, A., Cartellier, A., Hainaux, F., Lasheras, J., 2002. Effect of preferential concentration on the settling velocity of heavy particles in homogeneous isotropic turbulence. J. Fluid Mech. 468, 77-105. doi:10.1017/S0022112002001593.

Ayala, O., Parishani, H., Chen, L., Rosa, B., Wang, L.P., 2014. DNS of hydrodynamically interacting droplets in turbulent clouds: Parallel implementation and scalability analysis using 2D domain decomposition. Comp. Phys. Comm. 185, 3269-3290. doi $10.1016 /$ j.cpc.2014.09.005. 
Ayala, O., Rosa, B., Wang, L.P., Grabowski, W.W., 2008. Effects of turbulence on the geometric collision rate of sedimenting droplets. Part 1. Results from direct numerical simulation. New J. Phys. 10. doi:10.1088/1367-2630/10/7/07501.

Bosse, T., Kleiser, L., Meiburg, E., 2006. Small particles in homogeneous turbulence: Settling velocity enhancement by two-way coupling. Phys. Fluids 18. doi:10.1063/1.2166456.

Cadiergue, S., Michaux-Leblond, N., Belorgey, M., 1999. Settling velocity of a single heavy particle in a turbulent flow. C. R. Acad. Sci., Ser. II-B 327, 1379-1384. doi $10.1016 /$ S1287-4620(00)87508-0.

770 Chan, C.C., Fung, J.C.H., 1999. The change in setting velocity of inertial particles in cellular flow. Fluid Dyn. Res. 25, 257-273. doi $10.1016 /$ S0169-5983(98)00042-2.

Clift, R., Grace, J.R., Weber, M.E., 1978. Bubble, Drops, and Particles. Academic Press.

Crowe, C.T., Schwarzkopf, J.D., Sommerfeld, M., Tsuji, Y., 2011. Multiphase Flows with Droplets and Particles. CRC Press.

Cuthbertson, A., Ervine, D., 2007. Experimental study of fine sand particle settling in turbulent open channel flows over rough porous beds. J. Hydraul. Eng. 133, 905-916. doi 10.1061/(ASCE) 0733-9429(2007)133:8(905).

Davila, J., Hunt, J.C., 2001. Settling of small particles near vortices and in turbulence. J. Fluid Mech. 440, 117-145. doi 10.1017/S0022112001004694.

Dejoan, A., 2011. DNS experiments on the settling of heavy particles in homogeneous turbulence: two-way coupling and reynolds number effects. J. Phys.: Conf. Ser. 333. doi $10.1088 / 1742-6596 / 333 / 1 / 012006$

785 Eswaran, V., Pope, S.B., 1988. An examination of forcing in direct numerical simulations of turbulence. Comp. Fluids 16, 257-278. doi $10.1016 / 0045-7930$ (88)90013-8. 
Fokeer, S., Kingmana, S., Lowndes, I., Reynolds, A., 2004. Characterisation of the cross sectional particle concentration distribution in horizontal dilute flow conveying-a review. Chem. Eng. Process. 43, 677-691. doi $10.1016 /$ S0255-2701(03)00096-5.

Fung, J.C.H., 1993. Gravitational settling of particles and bubbles in homogeneous turbulence. J. Geophys. Res. 98, 20287-20297. doi:10.1029/93JC01845

Fung, J.C.H., 1997. Gravitational settling of small spherical particles in unsteady cellular flow fields. J. Aerosol Sci. 28, 753-787. doi $10.1016 /$ S0021-8502(96)00478-8.

Ghosh, S., Jonas, P.R., 2001. Some analytical calculations on the effect of turbulence on the settling and growth of cloud droplets. Geophys. Research Lett. 28, 3883-3886. doi 10.1029/2001GL013113.

Good, G.H., Ireland, P.J., Bewley, G.P., Bodenschatz, E., Collins, L.R., Warhaft, Z., 2014. Settling regimes of inertial particles in isotropic turbulence. J. Fluid Mech. 759. doi:10.1017/jfm.2014.602.

Grabowski, W.W., Wang, L.P., 2013. Growth of cloud droplets in a turbulent environment. Annu. Rev. Fluid Mech. 45, 293-324. doi:10.1146/annurev-fluid-011212-140750.

Ishihara, T., Gotoh, T., Kaneda, Y., 2009. Study of high-reynolds number isotropic turbulence by direct numerical simulation. Annu. Rev. Fluid Mech. 41, 165-180. doi 10.1146/annurev.fluid.010908.165203.

Kallio, G.A., Reeks, M.W., 1989. A numerical simulation of particle deposition in turbulent boundary layers. Int. J. Multiphase Flow 15, 433-446. doi $10.1016 / 0301-9322(89) 90012-8$,

Kawanisi, K., Shiozaki, R., 2008. Turbulent effects on the settling velocity of suspended sediment. J. Hydraul. Eng. 134, 261-266. doi $10.1061 /$ (ASCE) 0733-9429(2008) 134:2(261). 
Keshtpoor, M., Puleo, J.A., Shi, F., Ma, G., 2015. 3D numerical simulation of turbulence and sediment transport within a tidal inlet. Coastal Eng. 96, 13-26. doi $10.1016 / \mathrm{j}$. coastaleng.2014.10.009.

Lain, S., Sommerfeld, M., 2013. Characterisation of pneumatic conveying systems using the Euler/Lagrange approach. Powder Technol. 235, 764-782. doi: $10.1016 / j$. powtec.2012.11.029.

Li, Y., McLaughlin, J.B., Kontomaris, K., Portela, L., 2001. Numerical simulation of particle-laden turbulent channel flow. Phys. Fluids 13. doi $10.1063 / 1.1396846$

Lillo, F.D., Cecconi, F., Lacorata, G., Vulpiani, A., 2008. Sedimentation speed of inertial particles in laminar and turbulent flows. EPL 84. doi:10.1209/0295-5075/84/40005

Manton, M.J., 1974. On the motion of a small particle in the atmosphere. Boundary-Layer Meteorology 6, 487-504. doi:10.1007/BF02137681.

Maxey, M.R., 1987. The gravitational settling of aerosol particles in homogeneous turbulence and random flow fields. J. Fluid Mech. 174, 441-465. doi $10.1017 /$ S0022112087000193.

Maxey, M.R., Corrsin, S., 1986. Gravitational settling of aerosol particles in randomly oriented cellular flow fields. J. Atmos. Sci. 43, 1112-1134. doi $10.1175 / 1520-0469$ (1986) 043<1112: GSOAPI>2.0.C0;2.

Maxey, M.R., Riley, J.J., 1983. Equation of motion for a small rigid sphere in a nonuniform flow. Phys. Fluids 26, 883-889. doi 10.1063/1.864230.

Mei, R., 1994. Effect of turbulence on the particle settling velocity in the non-linear drag range. Int. J. Multiphase Flow 20, 273-284. 840 doi $10.1016 / 0301-9322(94) 90082-5$.

Mouri, H., Hori, A., Kawashima, Y., Hashimoto, K., 2012. Large-scale length that determines the mean rate of energy dissipation in turbulence. Physical Review E 86. doi:10.1103/PhysRevE.86.026309. 
Nielsen, P., 1993. Turbulence effects on the settling of suspended particles. J. Sedim. Petrol. 63, 835-838. doi:10.1306/D4267C1C-2B26-11D7-8648000102C1865D

Noh, Y., Kang, I.S., Herold, M., Raasch, S., 2006. Large eddy simulation of particle settling in the ocean mixed layer. Phys. Fluids 18. doi:10.1063/1.2337098

Orszag, S.A., Patterson, G.S., 1972. Numerical simulation of threedimensional homogeneous isotropic turbulence. Phys. Rev. Lett. 28, 76-79. doi:10.1103/PhysRevLett.28.76.

Papanicolaou, A., Elhakeem, M., Krallis, G., Prakash, S., Edinger, J., 2008. Sediment transport modeling reviewcurrent and future developments. J. Hydraulic Eng. 134, 1-14. doi 10.1061/(ASCE)0733-9429(2008)134:1(1).

Parishani, H., Ayala, O., Rosa, B., Wang, L.P., Grabowski, W.W., 2015. Effects of gravity on the acceleration and pair statistics of inertial particles in homogeneous isotropic turbulence. Phys. Fluids 27. doi 10.1063/1.4915121.

Pasquero, C., Provenzale, A., Spiegel, E.A., 2003. Suspension and fall of heavy particles in random two-dimensional flow. Phys. Rev. Lett. 91. doi:10.1103/PhysRevLett.91.054502

Rosa, B., Parishani, H., Ayala, O., Grabowski, W.W., Wang, L.P., 2013. Kinematic and dynamic collision statistics of cloud droplets from high-resolution simulations. New J. Phys. 15. doi:10.1088/1367-2630/15/4/045032.

Rosa, B., Parishani, H., Ayala, O., Wang, L.P., 2015. Effects of forcing time scale on the simulated turbulent flows and turbulent collision statistics of inertial particles. Phys. Fluids 27. doi:10.1063/1.4906334.

Ruiz, J., Macias, D., Peters, F., 2004. Turbulence increases the average settling velocity of phytoplankton cells. Proc. Natl. Acad. Sci. USA 101, 17720-17724. doi:10.1073/pnas. 0401539101 . 
Siebert, H., Lehmann, K., Wendisch, M., 2006. Observations of small-scale turbulence and energy dissipation rates in the cloudy boundary layer. J. Atmos. Sci. 63, 1451-1466. doi:10.1175/JAS3687.1.

Smoot, L., 2013. Pulverized-Coal Combustion and Gasification: Theory and Applications for Continuous Flow Processes. Springer US.

Squires, K.D., Eaton, J.K., 1991. Preferential concentration of particles by turbulence. Phys. Fluids A 3, 1169-1178. doi $10.1063 / 1.858045$.

Stout, J.E., Arya, S.P., Genikhovich, E.L., 1995. The effect of nonlinear drag on the motion and settling velocity of heavy particles. J. Atmos. Sci. 52, 3836-3848. doi 10.1175/1520-0469(1995)052<3836:TEONDO>2.0.C0;2.

Sullivan, N.P., Mahalingam, S., Kerr, R.M., 1994. Deterministic forcing of homogeneous isotropic turbulence. Phys. Fluids 6. doi:10.1063/1.868274.

Sundaram, S., Collins, L.R., 1997. Collision statistics in an isotropic particleladen turbulent suspension. part 1. direct numerical simulations. J. Fluid Mech. 335, 75-109. doi 10.1017/S0022112096004454

Tooby, P.F., Wick, G.L., Isaacs, J.D., 1977. The motion of a small sphere in a rotating velocity field: A possible mechanism for suspending particles in turbulence. J. Geophys. Res. 82, 2096-2100. doi 10.1029/JC082i015p02096

Tsidulko, M., Krichak, S.O., Alpert, P., Kakaliagou, O., Kallos, G., Papadopoulos, A., 2002. Numerical study of a very intensive eastern mediterranean dust storm, 13-16 march 1998. J. Geophys. Res. 107. doi 10.1029/2001JD001168.

Vilela, R.D., Motter, A.E., 2007. Can aerosols be trapped in open flows? Phys. Rev. Lett. 101. doi:10.1103/PhysRevLett.99.264101.

Wang, L.P., Ayala, O., Y.Xue, Grabowski, W.W., 2006. Comments on "Droplets to Drops by Turbulent Coagulation". J. Atmos. Sci. 63, 2397-2401. doi:10.1175/JAS3750.1. 
Wang, L.P., Maxey, M.R., 1993. Settling velocity and concentration distribution of heavy particles in homogeneous isotropic turbulence. J. Fluid Mech. 256, 26-68. doi $10.1017 /$ S0022112093002708.

Yang, C.Y., Lei, U., 1998. The role of turbulent scales in the settling velocity of heavy particles in homogeneous isotropic turbulence. J. Fluid Mech. 371, 179-205. doi:10.1017/S0022112098002328.

Yang, T.S., Shy, S.S., 2003. The settling velocity of heavy particles in an aqueous near-isotropic turbulence. Phys. Fluids 15, 868-880. doi 10.1063/1.1557526

Yang, T.S., Shy, S.S., 2005. Two-way interaction between solid particles and homogeneous air turbulence: particle settling rate and turbulence modification measurements. J. Fluid Mech. 526, 171-216. doi 0.1017/S002211200400286

Zhou, H., Flamant, G., Gauthier, D., 2004. DEM-LES of coal combustion in a bubbling fluidized bed. Part I: gas-particle turbulent flow structure. Chem. Eng. Sci. 59, 4193-4203. doi $10.1016 /$ j.ces.2004.01.069.

Zhou, Q., Cheng, N.S., 2009. Experimental investigation of single particle settling in turbulence generated by oscillating grid. Chem. Eng. J. 149, 289-300. doi:10.1016/j.cej.2008.11.004.

Zhou, Y., Wexler, A.S., Wang, L.P., 2001. Modelling turbulent col915 lision of bidisperse inertial particles. J. Fluid Mech. 433, 77-104.
doi:10.1017/S0022112000003372. 